\title{
On Nonlinear Oscillations of a Near-Autonomous Hamiltonian System in the Case of Two Identical Integer or Half-Integer Frequencies
}

\author{
O. V. Kholostova
}

This paper examines the motion of a time-periodic Hamiltonian system with two degrees of freedom in a neighborhood of trivial equilibrium. It is assumed that the system depends on three parameters, one of which is small; when it has zero value, the system is autonomous. Consideration is given to a set of values of the other two parameters for which, in the autonomous case, two frequencies of small oscillations of the linearized equations of perturbed motion are identical and are integer or half-integer numbers (the case of multiple parametric resonance). It is assumed that the normal form of the quadratic part of the Hamiltonian does not reduce to the sum of squares, i.e., the trivial equilibrium of the system is linearly unstable. Using a number of canonical transformations, the perturbed Hamiltonian of the system is reduced to normal form in terms through degree four in perturbations and up to various degrees in a small parameter (systems of first, second and third approximations). The structure of the regions of stability and instability of trivial equilibrium is investigated, and solutions are obtained to the problems of the existence, number, as well as (linear and nonlinear) stability of the system's periodic motions analytic in fractional or integer powers of the small parameter. For some cases, conditionally periodic motions of the system are described. As an application, resonant periodic motions of a dynamically symmetric satellite modeled by a rigid body are constructed in a neighborhood of its steady rotation (cylindrical precession) on a weakly elliptic orbit and the problem of their stability is solved.

Keywords: multiple parametric resonance, method of normal forms, stability, nonlinear oscillations, periodic motions, dynamically symmetric satellite, cylindrical precession

Received February 01, 2021

Accepted March 19, 2021

This research was supported by the grant of the Russian Science Foundation (project 19-11-00116) and was carried out at the Moscow Aviation Institute (National Research University).

Olga V. Kholostova

kholostova_o@mail.ru

Moscow Aviation Institute (National Research University)

Volokolamskoe sh. 4, GSP-3, A-80, Moscow, 125993 Russia 


\section{Introduction}

The study of the cases of multiple parametric resonances in near-autonomous, time-periodic linear Hamiltonian systems in a neighborhood of trivial equilibrium has been started relatively recently in the work of A. P. Markeev [1-3]. A systematization of all such cases was performed, an algorithm for constructing instability regions (regions of parametric resonance) was proposed, and the possibility of existence of several (from one to three) regions of instability of equilibrium in a neighborhood of the resonant point of the parameter plane of the system was shown for the first time.

A more detailed analysis of the structure of stability and instability regions in a number of cases of multiple parametric resonances was carried out in [4-7], whereas this paper deals with problems of the existence, number and stability of nonlinear resonant periodic motions of the system and problems of the existence of conditionally periodic motions [6].

This paper continues the series of studies undertaken previously. It is assumed that the system depends on three parameters, one of which is small such that, when it has zero value, the system is autonomous. An analysis is made of the set of values of the other two parameters for which, in the autonomous case, two frequencies of small oscillations of linearized equations of perturbed motion are identical and are integer or half-integer numbers. It is also assumed that the normalized quadratic part of the Hamiltonian of perturbed motion does not reduce to the sum of squares, i.e., the trivial equilibrium of the linearized system is unstable.

It should be noted that the above-mentioned case of two identical frequencies occurs rather often in autonomous systems with two degrees of freedom and has been well studied both from a theoretical point of view and in relation to specific mechanical systems [8-16]. In this paper, this case has been generalized to a near-autonomous and time-periodic system. In systems of first, second and third approximations in a small parameter, the structure of the regions of stability and instability of trivial equilibrium is studied. Solutions are found to the problems of the existence, number and (linear and nonlinear) stability of the system's periodic motions analytic in fractional or integer powers of the small parameter. For some cases, conditionally periodic motions of the system are described.

As an application to the theoretical results obtained, resonant periodic motions of a dynamically symmetric satellite modeled by a rigid body are constructed in a neighborhood of its steady rotation (cylindrical precession) on a weakly elliptic orbit and the problem of their stability is solved.

\section{Formulation of the problem. Transformation of the Hamiltonian}

Consider the motions of a two-degree-of-freedom Hamiltonian system $2 \pi$-periodic in time. Suppose that the Hamiltonian $H\left(q_{j}, p_{j}, t ; \alpha, \beta, \varepsilon\right)$ of the system depends on parameters $\alpha$ and $\beta$ and on a small parameter $\varepsilon(0<\varepsilon \ll 1)$, and let the system be autonomous when $\varepsilon=0$. It is assumed that the origin of coordinates $q_{j}=0, p_{j}=0(j=1,2)$ of the phase space is the equilibrium point of the system, and in its neighborhood the Hamiltonian is analytic in its arguments and is represented as the series

$$
\begin{aligned}
H & =H_{2}+H_{4}+H_{6}+\ldots, \\
H_{2} & =H_{20}\left(q_{j}, p_{j}, \alpha, \beta\right)+\varepsilon H_{21}\left(q_{j}, p_{j}, \alpha, \beta, t\right)+\varepsilon^{2} H_{22}\left(q_{j}, p_{j}, \alpha, \beta, t\right)+\ldots, \\
H_{4} & =H_{40}\left(q_{j}, p_{j}, \alpha, \beta\right)+\varepsilon H_{41}\left(q_{j}, p_{j}, \alpha, \beta, t\right)+\varepsilon^{2} H_{42}\left(q_{j}, p_{j}, \alpha, \beta, t\right)+\ldots,
\end{aligned}
$$


which contains only terms of even degrees in $q_{j}$ and $p_{j}$. Here, $H_{l k}$ are homogeneous forms of degree $l$ in $q_{j}$ and $p_{j}$ with coefficients constant (for $k=0$ ) or $2 \pi$-periodic in $t$ (for $k=1,2, \ldots$ ).

We assume that the value, averaged over a period (in time), of the function $H_{21}$ is zero; this condition is fulfilled rather often in problems of classical and celestial mechanics.

Suppose that in the autonomous case $(\varepsilon=0)$ for some values $\alpha=\alpha_{*}, \beta=\beta_{*}$ the frequencies $\omega_{1}$ and $\omega_{2}$ of small oscillations of linearized (in a neighborhood of trivial equilibrium) equations of motion of the system satisfy the relation $2 \omega_{1}=2 \omega_{2}=n$ ( $n$ being a natural number), and for these frequency values the quadratic part $H_{20}$ does not reduce to the sum of squares. Its normal form is (we keep the previous notation for the variables)

$$
H_{20}=\frac{1}{2}\left(p_{1}^{2}+p_{2}^{2}\right)+\frac{n}{2}\left(q_{1} p_{2}-p_{1} q_{2}\right) .
$$

In this case, the trivial equilibrium of the autonomous linear system is unstable.

Next, we will explore the nonlinear oscillations of the system with the Hamiltonian (2.1) near its trivial equilibrium for the parameter values from a small neighborhood of the resonant point $\varepsilon=0, \alpha=\alpha_{*}, \beta=\beta_{*}$ of the three-dimensional parameter space. The structure of the regions of stability and instability of trivial equilibrium will be studied, and the problems of the existence, number and (linear and nonlinear) stability of the system's periodic motions analytic in fractional or integer powers of the small parameter will be solved. For some special cases, conditionally periodic motions of the system will be described.

We note that the double parametric resonance of this type (and that of other types) in the Hamiltonian system under consideration can arise only in the case where the autonomous part of the Hamiltonian depends on two parameters. For example, resonance detunings of two eigenfrequencies of small oscillations can serve as such parameters. By taking account of these parameters, which are chosen in the small neighborhood of the resonant point, and by adding the small parameter from the nonautonomous perturbing part, one can obtain a complete description of the bifurcation properties of the system.

Suppose the quadratic part $H_{20}$ has already been reduced to the form (2.2). At the first stage, using a number of canonical transformations, we simplify (normalize) the structure of the Hamiltonian in the second- and fourth-degree terms in perturbations.

We will perform the analysis in a small neighborhood of order $\varepsilon$ of the resonant point given by the formulae

$$
\alpha=\alpha_{*}+\varepsilon \nu_{1}, \quad \beta=\beta_{*}+\varepsilon \mu_{1}+\varepsilon^{2} \mu_{2}+\varepsilon^{3} \mu_{3}+\varepsilon^{4} \mu_{4}+\ldots,
$$

where $\nu_{1}$ and $\mu_{k}$ are constants. The "truncated" form of representation of the parameter $\alpha$ does not restrict the generality of the investigation since the coefficient $\nu_{1}$ can, in turn, consist of terms of different powers in $\varepsilon$. However, the adopted representation make it possible to find in the next sections in a clear and more convenient way equations describing the boundaries of the regions of parametric resonance in which the dependences $\mu_{1}=\mu_{1}\left(\nu_{1}\right), \mu_{2}=\mu_{2}\left(\nu_{1}\right), \ldots$ are sequentially obtained.

We first make a near-identity canonical transformation $q_{j}, p_{j} \rightarrow q_{j}^{\prime}, p_{j}^{\prime}(j=1,2)$ (differing from an identity transformation by third-degree terms in perturbations), which normalizes the form $H_{40}$ of degree four in the autonomous part of the Hamiltonian. The calculations performed, for example, using the Deprit-Hori method [10] yield ( $\gamma_{k}=$ const)

$$
K_{40}=\gamma_{1}\left(q_{1}^{\prime 2}+q_{2}^{\prime 2}\right)^{2}+\gamma_{2}\left(q_{1}^{\prime 2}+q_{2}^{\prime 2}\right)\left(q_{1}^{\prime} p_{2}^{\prime}-p_{1}^{\prime} q_{2}^{\prime}\right)+\gamma_{3}\left(q_{1}^{\prime} p_{2}^{\prime}-p_{1}^{\prime} q_{2}^{\prime}\right)^{2} .
$$

Since the explicit form of the transformation is cumbersome, we do not present it here. Next, we assume that $\gamma_{1} \neq 0$. 
The further univalent canonical change of variables [2]

$$
\begin{array}{ll}
q_{1}^{\prime}=x_{1} \cos \left(\frac{n}{2} t\right)-x_{2} \sin \left(\frac{n}{2} t\right), & q_{2}^{\prime}=x_{1} \sin \left(\frac{n}{2} t\right)+x_{2} \cos \left(\frac{n}{2} t\right), \\
p_{1}^{\prime}=X_{1} \cos \left(\frac{n}{2} t\right)-X_{2} \sin \left(\frac{n}{2} t\right), & p_{2}^{\prime}=X_{1} \sin \left(\frac{n}{2} t\right)+X_{2} \cos \left(\frac{n}{2} t\right),
\end{array}
$$

transforms the quadratic form (2.2) to

$$
K_{20}=\frac{1}{2} X_{1}^{2}+\frac{1}{2} X_{2}^{2} .
$$

We note that this change of variables preserves, up to notation, the form (2.4).

We now consider the nonautonomous quadratic part of the Hamiltonian. Taking (2.3) into account, we expand the form $H_{2}$ from (2.1) as a series in powers of $\varepsilon$ :

$$
\begin{aligned}
H_{2} & =\widetilde{H}_{20}+\varepsilon \widetilde{H}_{21}+\varepsilon^{2} \widetilde{H}_{22}+\ldots, \\
\widetilde{H}_{20} & =H_{20}, \quad \widetilde{H}_{21}=\left.H_{21}\right|_{*}+\left.\frac{\partial H_{20}}{\partial \alpha}\right|_{*} \nu_{1}+\left.\frac{\partial H_{20}}{\partial \beta}\right|_{*} \mu_{1}, \ldots
\end{aligned}
$$

Here, the symbol $*$ means that the calculation is performed for $\alpha=\alpha_{*}, \beta=\beta_{*}$.

The partial derivatives in the second and third terms in $\widetilde{H}_{21}$ are quadratic forms with constant coefficients. We write them as

$$
\begin{aligned}
\left.\frac{\partial H_{20}}{\partial \alpha}\right|_{*} & =a_{1} q_{1}^{2}+a_{2} q_{2}^{2}+a_{3} p_{1}^{2}+a_{4} p_{2}^{2}+ \\
& +a_{5} q_{1} q_{2}+a_{6} q_{1} p_{1}+a_{7} q_{1} p_{2}+a_{8} q_{2} p_{1}+a_{9} q_{2} p_{2}+a_{10} p_{1} p_{2} \\
\left.\frac{\partial H_{20}}{\partial \beta}\right|_{*} & =b_{1} q_{1}^{2}+b_{2} q_{2}^{2}+b_{3} p_{1}^{2}+b_{4} p_{2}^{2}+ \\
& +b_{5} q_{1} q_{2}+b_{6} q_{1} p_{1}+b_{7} q_{1} p_{2}+b_{8} q_{2} p_{1}+b_{9} q_{2} p_{2}+b_{10} p_{1} p_{2} .
\end{aligned}
$$

We represent the nonautonomous quadratic form $\left.H_{21}\right|_{*}$ as a Fourier series

$$
\left.H_{21}\right|_{*}=\sum_{k=1}^{\infty}\left[D_{k}\left(q_{j}, p_{j}\right) \cos k t+E_{k}\left(q_{j}, p_{j}\right) \sin k t\right],
$$

where $D_{k}\left(q_{j}, p_{j}\right)$ and $E_{k}\left(q_{j}, p_{j}\right)$ are quadratic forms of variables $q_{j}, p_{j}(j=1,2)$ with constant coefficients $d_{k l}$ and $e_{k l}$. In this case, the terms with $d_{k l}$ and $e_{k l}$ contain the same combination of variables $q_{j}, p_{j}$ as the terms with $a_{l}$ or $b_{l}$ in the formula (2.8).

We make a change of variables (2.5) and then, using the Deprit-Hori method, a $T$-periodic (in time) canonical near-identity transformation (differing from an identity transformation by terms of order $\varepsilon$ and higher), which normalizes the form $H_{2}$ in terms up to the required order in $\varepsilon$. Here, $T=4 \pi$ and $T=2 \pi$, respectively, for odd and even values of $n$ in resonant relation. As a result, the time in the normalized part is eliminated, and the terms with constant coefficients acquire a simpler and more symmetric structure.

Keeping the notation $x_{j}, X_{j}(j=1,2)$ for the variables, we represent the transformed quadratic form as

$$
K_{2}=K_{20}+\varepsilon K_{21}\left(x_{j}, X_{j}\right)+\ldots+\varepsilon^{k} K_{2 k}\left(x_{j}, X_{j}\right)+\varepsilon^{k+1} \widetilde{K}_{2}\left(x_{j}, X_{j}, t ; \varepsilon\right),
$$

where the term $K_{20}$ is defined in (2.6). The explicit form of $K_{21}, \ldots, K_{2 k}$ for the system of the $k$ th approximation $(k=1,2,3)$ will be represented in the next sections. The last term in $(2.10)$ is $T$-periodic in $t$. 
REMARK. In the case of two identical integer frequencies the system has third-order resonances; however, if the Hamiltonian has no third-degree terms in perturbations (which is assumed in this study), these resonances are insignificant. Conversely, if the Hamiltonian does have third-degree terms, then, after their normalization, terms with resonant harmonics remain, resulting in a more complex structure of the normalized Hamiltonians. We obtain a different problem which requires a separate study going beyond the scope of this work.

In the case of two identical half-integer frequencies there are no third-order resonances, and using an additional canonical change of variables, the third-degree terms in perturbations (if any) can be eliminated. This case does not differ from that considered in this paper.

\section{A model system of first approximation}

\subsection{Construction of a model Hamiltonian}

Simplifying the structure of the terms of order one in $\varepsilon$ using the Deprit-Hori method, we obtain the following form $K_{21}$ :

$$
\begin{aligned}
K_{21} & =\frac{1}{2}\left(c_{1}+c_{2}+c_{0}\right) x_{1}^{2}+\frac{1}{2}\left(c_{1}+c_{2}-c_{0}\right) x_{2}^{2}+d_{0} x_{1} x_{2}+\frac{1}{2}\left(c_{7}-c_{8}\right)\left(x_{1} X_{2}-x_{2} X_{1}\right), \\
c_{j} & =a_{j} \nu_{1}+b_{j} \mu_{1}(j \neq 0), \quad c_{0}=\frac{1}{2}\left(e_{n 5}+d_{n 1}-d_{n 2}\right), \quad d_{0}=\frac{1}{2}\left(d_{n 5}-e_{n 1}+e_{n 2}\right) .
\end{aligned}
$$

Another canonical transformation of the form

$$
\begin{array}{ll}
x_{1}=\widetilde{x}_{1} \cos \delta-\widetilde{x}_{2} \sin \delta, & X_{1}=\widetilde{X}_{1} \cos \delta-\widetilde{X}_{2} \sin \delta, \\
x_{2}=\widetilde{x}_{1} \sin \delta+\widetilde{x}_{2} \cos \delta, & X_{2}=\widetilde{X}_{1} \sin \delta+\widetilde{X}_{2} \cos \delta
\end{array}
$$

eliminates in (3.1) the term containing the product $x_{1} x_{2}$. This yields

$$
\widetilde{K}_{21}=\frac{1}{2}\left(c_{1}+c_{2}+\sigma \widetilde{d}\right) \widetilde{x}_{1}^{2}+\frac{1}{2}\left(c_{1}+c_{2}-\sigma \widetilde{d}\right) \widetilde{x}_{2}^{2}+\frac{1}{2}\left(c_{7}-c_{8}\right)\left(\widetilde{x}_{1} \widetilde{X}_{2}-\widetilde{x}_{2} \widetilde{X}_{1}\right)
$$

Here we have introduced the notation

$$
\delta=\frac{1}{2} \arctan \frac{d_{0}}{c_{0}}, \quad \sigma=\operatorname{sign} c_{0}, \quad \widetilde{d}=\sqrt{c_{0}^{2}+d_{0}^{2}} .
$$

The transformation (3.3) preserves, up to notation of variables, the forms $K_{20}$ and $H_{40}$.

We now consider the complete nonlinear system. Let us pass to a small (of order $\varepsilon^{1 / 2}$ ) neighborhood of trivial equilibrium using the formulae

$$
\widetilde{x}_{j}=\varepsilon^{1 / 2} y_{j}, \quad \tilde{X}_{j}=\varepsilon^{1 / 2} Y_{j} \quad(j=1,2) .
$$

The transformed complete Hamiltonian of the system can be written as

$$
\Gamma^{(1)}=\Gamma^{(10)}+O\left(\varepsilon^{2}\right), \quad \Gamma^{(10)}=\Gamma_{20}+\varepsilon\left(\Gamma_{21}+\Gamma_{40}\right) .
$$

Here, $\Gamma_{20}, \Gamma_{40}$ and $\Gamma_{21}$ are, respectively, the forms $\widetilde{K}_{20}, \widetilde{K}_{40}$ and $\widetilde{K}_{21}$, in which $\widetilde{x}_{j}$ and $\widetilde{X}_{j}$ have been replaced by $y_{j}$ and $Y_{j}$. The term $O\left(\varepsilon^{2}\right)$ is $T$-periodic in $t$. 
We will call the approximate Hamiltonian $\Gamma^{(10)}$ and the corresponding autonomous system a model Hamiltonian and a model system of first approximation, respectively.

Note that, in the constructed model system, the case $\sigma=-1$ reduces to the case $\sigma=1$ if one interchanges indices 1 and 2 in the variables $y_{j}, Y_{j}$ and changes the sign of the coefficient in front of the last combination in the form $\Gamma_{21}$ and the sign of the coefficient $\gamma_{2}$ in the form $\Gamma_{40}$. However, it follows from the results obtained below that the qualitative properties of the system do not depend on the signs of the above-mentioned coefficients. Therefore, in what follows we assume that $\sigma=1$.

\subsection{Stability of the trivial equilibrium of the system}

In investigating the stability of the equilibrium points of the autonomous model systems under study, we consider the quadratic part of the Hamiltonian of the motion perturbed in the neighborhood of equilibrium. By virtue of the Lyapunov stability theorem, a sufficient condition for the stability of equilibrium is the sign definiteness of this quadratic part. Necessary conditions for stability are obtained in the analysis of the characteristic equation of the linearized equations of perturbed motion, which has the form

$$
\lambda^{4}+a \lambda^{2}+b=0 .
$$

If the conditions

$$
a>0, \quad b>0, \quad d=a^{2}-4 b>0
$$

hold, then the roots of equation (3.6) are purely imaginary, and the equilibrium of the model system under study is linearly stable. When the sign of at least one of inequalities (3.7) is reversed, equation (3.6) has roots with positive real parts, and this equilibrium is linearly and nonlinearly unstable.

Consider the quadratic part $\Gamma_{20}+\varepsilon \Gamma_{21}$ of the Hamiltonian of the model system of first approximation. The coefficients of the corresponding characteristic equation and its discriminant are

$$
a=2\left(c_{1}+c_{2}\right) \varepsilon+O\left(\varepsilon^{2}\right), \quad b=\left[\left(c_{1}+c_{2}\right)^{2}-\widetilde{d}^{2}\right] \varepsilon^{2}+O\left(\varepsilon^{3}\right), \quad d=4 \widetilde{d}^{2} \varepsilon^{2}+O\left(\varepsilon^{3}\right) .
$$

For sufficiently small values of $\varepsilon$ the necessary conditions for stability (3.7) of the trivial equilibrium of the system reduce to the inequality

$$
c_{1}+c_{2}>\tilde{d}
$$

under the condition $\widetilde{d} \neq 0$. If $c_{1}+c_{2}<\widetilde{d}$, then for all $\widetilde{d} \geqslant 0$ this equilibrium is unstable.

Inequality (3.9) is for all $\widetilde{d} \geqslant 0$ also a sufficient condition for the stability of trivial equilibrium, since in this case the quadratic part of the Hamiltonian is positive definite.

The boundary $c_{1}+c_{2}=\widetilde{d}$ between the regions of stability and instability, with the expressions for $c_{j}$ from (3.2) taken into account, corresponds to a straight line in the parameter plane $(\alpha, \beta)$, which divides the neighborhood of the resonant point into two half-planes, in one of which the trivial equilibrium of the model system is stable, and in the other, unstable.

When $\widetilde{d}=0$ and $c_{1}+c_{2}=0$, the pattern of stability of the trivial equilibrium of the system in this approximation in $\varepsilon$ is not defined. In this case, it is necessary to take into account terms of the next approximations in $\varepsilon$. This will be done in Section 4 . 


\subsection{Nontrivial equilibria of the model system}

Equating to zero the partial derivatives of the model Hamiltonian $\Gamma^{(10)}$ of (3.5) in the variables $y_{j}, Y_{j}(j=1,2)$, we obtain an algebraic system of equations to define nontrivial equilibrium points of the model system.

First let $\widetilde{d} \neq 0$. Analysis of these equations shows that under the condition $\gamma_{1}\left(c_{1}+c_{2}+\widetilde{d}\right)<0$ the model system has a pair of equilibria given by the relations

$$
\begin{gathered}
y_{2}=0, \quad Y_{1}=0, \quad y_{1}=y_{10}+O(\varepsilon), \\
y_{10}^{2}=-\frac{c_{1}+c_{2}+\tilde{d}}{4 \gamma_{1}}, \quad Y_{2}=-\varepsilon\left[\frac{c_{7}-c_{8}}{2}+\gamma_{2} y_{10}^{2}\right] y_{10}+O\left(\varepsilon^{2}\right) .
\end{gathered}
$$

If $\gamma_{1}\left(c_{1}+c_{2}-\widetilde{d}\right)<0$, then there is another pair of equilibria for which

$$
\begin{gathered}
y_{1}=0, \quad Y_{2}=0, \quad y_{2}=y_{20}+O(\varepsilon), \\
y_{20}^{2}=-\frac{c_{1}+c_{2}-\widetilde{d}}{4 \gamma_{1}}, \quad Y_{1}=\varepsilon\left[\frac{c_{7}-c_{8}}{2}+\gamma_{2} y_{20}^{2}\right] y_{20}+O\left(\varepsilon^{2}\right) .
\end{gathered}
$$

In considering the corresponding characteristic equations of the linearized equations of perturbed motion, we find that in the existence region the first pair of equilibria is always unstable. When $\gamma_{1}<0$, the second pair is also unstable. When $\gamma_{1}>0$, necessary (and simultaneously sufficient) stability conditions are fulfilled in the region of existence of the second pair.

These results are illustrated in Fig. 1a, b for the cases $\gamma_{1}>0$ and $\gamma_{1}<0$, respectively. Here it is assumed that $a_{1}+a_{2}>0$ and $b_{1}+b_{2}>0$; other variations of the signs of these quantities can be considered in a similar way. The parameter plane $x=\alpha-\alpha_{*}, y=\beta-\beta_{*}$ is shown for a fixed value of $\varepsilon$.

The neighborhood of the resonant point $(0,0)$ is divided into three regions by the parallel straight lines $g_{-}$and $g_{+}$given, respectively, by the equations $c_{1}+c_{2}=-\widetilde{d}$ and $c_{1}+c_{2}=\widetilde{d}$, or

$$
\left(a_{1}+a_{2}\right) x+\left(b_{1}+b_{2}\right) y=\mp \varepsilon \widetilde{d} .
$$

The straight line $g_{+}$is also the boundary of the stability region of trivial equilibrium of the system. This region is colored in Fig. 1.

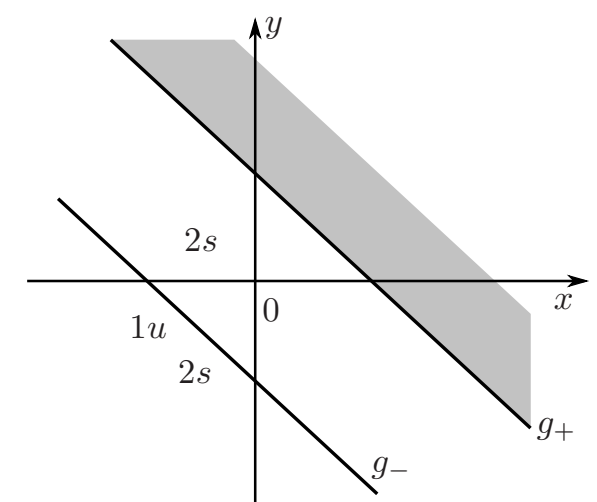

(a)

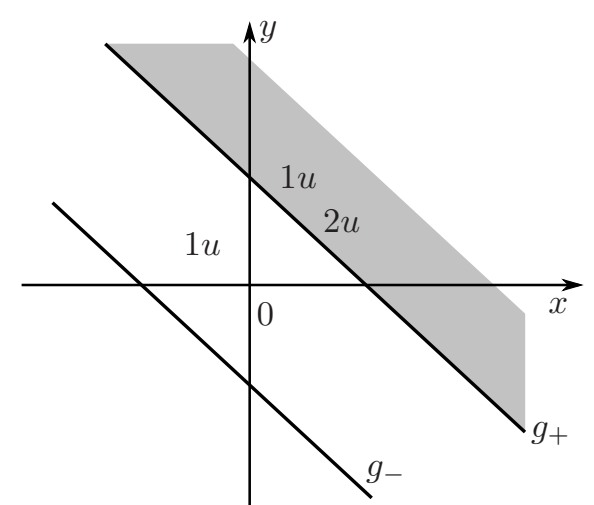

(b)

Fig. 1. The stability pattern for the first-approximation system. 
The regions where the equilibria (3.10) and (3.11) exist are denoted as 1 and 2, respectively; the symbols $s$ or $u$ mean that the equilibria in this region are stable or unstable.

When $\widetilde{d}=0$, the model system has (under the condition $\gamma_{1}\left(c_{1}+c_{2}\right)<0$ ) a family of unstable equilibria for which the pairs of points $\left(y_{10}, y_{20}\right)$ make up a circle

$$
y_{10}^{2}+y_{20}^{2}=-\frac{c_{1}+c_{2}}{4 \gamma_{1}} .
$$

In this case, the solutions (3.10) and (3.11) are unstable complex singular points of the system; they correspond to four points of the above-mentioned circle.

We note that in the case $\widetilde{d}=0$ qualitative changes occur in the properties of the model system. A cyclic coordinate appears in the system, and the motion pattern is determined by the properties of the reduced system with one degree of freedom. This is discussed in detail below in Section 7.

Finally, when the conditions $\widetilde{d}=0$ and $c_{1}+c_{2}=0$ are satisfied simultaneously, all equilibria of the system shrink to the origin. In this case, in a neighborhood of the straight line given by the equation $c_{1}+c_{2}=0$, it is necessary to investigate the system of second approximation.

\section{A model system of second approximation}

\subsection{Construction of a model Hamiltonian}

Now let the conditions $c_{1}+c_{2}=0$ and $\widetilde{d}=0$ hold simultaneously. Let us construct a model Hamiltonian of second approximation, taking into account terms of order $\varepsilon^{2}$ in the quadratic (in perturbations) part of the Hamiltonian.

Under the above conditions the quadratic form (3.4) becomes

$$
K_{21}=\frac{1}{2}\left(\widehat{c}_{7}-\widehat{c}_{8}\right)\left(x_{1} X_{2}-x_{2} X_{1}\right),
$$

where $\widehat{c}_{7}$ and $\widehat{c}_{8}$ are the coefficients $c_{7}$ and $c_{8}$ calculated for $c_{1}+c_{2}=0$, i.e., for $\mu_{1}=\mu_{1 *}=$ $=-\left(a_{1}+a_{2}\right) \nu_{1} /\left(b_{1}+b_{2}\right)($ see $(3.2))$.

The terms $\widetilde{H}_{22}$ from (2.7) are normalized in a similar way. The transformed form $K_{22}$ reads

$$
\begin{aligned}
K_{22} & =\frac{1}{2}\left[\left(b_{1}+b_{2}\right) \mu_{2}+d_{2}^{(1)}+e_{0}\right] x_{1}^{2}+\frac{1}{2}\left[\left(b_{1}+b_{2}\right) \mu_{2}+d_{2}^{(1)}-e_{0}\right] x_{2}^{2}+ \\
& +\frac{1}{2}\left(\left(b_{7}-b_{8}\right) \mu_{2}+d_{2}^{(2)}\right)\left(x_{1} X_{2}-x_{2} X_{1}\right) .
\end{aligned}
$$

Here, $d_{2}^{(j)}(j=1,2)$ are some polynomials of degree two in $\nu_{1}$, and $e_{0}=$ const. As in the first-approximation system, one can assume without loss of generality that $e_{0} \geqslant 0$.

Let us pass to the $\varepsilon$-neighborhood of the origin of the phase space using the formulae

$$
x_{j}=\varepsilon y_{j}, \quad X_{j}=\varepsilon Y_{j} \quad(j=1,2) .
$$

The transformed Hamiltonian takes the form

$$
\Gamma^{(2)}=\Gamma^{(20)}+O\left(\varepsilon^{3}\right), \quad \Gamma^{(20)}=\Gamma_{20}+\varepsilon \widetilde{\Gamma}_{21}+\varepsilon^{2}\left(\widetilde{\Gamma}_{22}+\Gamma_{40}\right) .
$$

Here, like the previous Hamiltonian, $\widetilde{\Gamma}_{21}$ and $\widetilde{\Gamma}_{22}$ are the forms (4.1) and (4.2) under the transformatiion $x_{j}=y_{j}, X_{j}=Y_{j}(j=1,2)$. The term $O\left(\varepsilon^{3}\right)$ is $T$-periodic in $t$.

We call the approximate Hamiltonian $\Gamma^{(20)}$ and the corresponding system a model Hamiltonian and a model system of second approximation, respectively. 


\subsection{Stability of the trivial equilibrium of the system}

For the characteristic equation of the linear system with the Hamiltonian $\Gamma_{20}+\varepsilon \Gamma_{21}+\varepsilon^{2} \Gamma_{22}$ we obtain

$$
\begin{aligned}
& a=2\left[\left(b_{1}+b_{2}\right) \mu_{2}+d_{2}^{(1)}+\frac{1}{4}\left(\widehat{c}_{7}-\widehat{c}_{8}\right)^{2}\right] \varepsilon^{2}+O\left(\varepsilon^{3}\right), \\
& b=\left[\left(\left(b_{1}+b_{2}\right) \mu_{2}+d_{2}^{(1)}-\frac{1}{4}\left(\widehat{c}_{7}-\widehat{c}_{8}\right)^{2}\right)^{2}-e_{0}^{2}\right] \varepsilon^{4}+O\left(\varepsilon^{5}\right), \\
& d=4\left[\left(\widehat{c}_{7}-\widehat{c}_{8}\right)^{2}\left(\left(b_{1}+b_{2}\right) \mu_{2}+d_{2}^{(1)}\right)+e_{0}^{2}\right] \varepsilon^{4}+O\left(\varepsilon^{5}\right) .
\end{aligned}
$$

Analyzing these expressions, we find that in the case where the quantities

$$
\left(b_{1}+b_{2}\right) \mu_{2}+d_{2}^{(1)}-\frac{1}{4}\left(\widehat{c}_{7}-\widehat{c}_{8}\right)^{2} \text { and } e_{0}
$$

do not vanish simultaneously, the trivial equilibrium of the linear system is stable when the condition

$$
\left(b_{1}+b_{2}\right) \mu_{2}+d_{2}^{(1)}>\frac{1}{4}\left(\widehat{c}_{7}-\widehat{c}_{8}\right)^{2}+e_{0}
$$

holds and when the following conditions are satisfied simultaneously:

$$
\begin{gathered}
\left(\widehat{c}_{7}-\widehat{c}_{8}\right)^{2}>2 e_{0}, \\
-\frac{e_{0}^{2}}{\left(\widehat{c}_{7}-\widehat{c}_{8}\right)^{2}}<\left(b_{1}+b_{2}\right) \mu_{2}+d_{2}^{(1)}<\frac{1}{4}\left(\widehat{c}_{7}-\widehat{c}_{8}\right)^{2}-e_{0} .
\end{gathered}
$$

In this case, inequality (4.5) is a necessary and simultaneously sufficient condition for stability, and inequalities (4.6) and (4.7) are only necessary conditions for stability of the trivial equilibrium of the model system. These conditions impose a restriction on the value of $\mu_{2}$.

If both quantities in (4.4) vanish, then the stability pattern of zero equilibrium in this approximation in $\varepsilon$ is not defined.

\subsection{Nontrivial equilibria of the model system}

In the model system of second approximation, as in the first-approximation system, there can exist up to two pairs of nontrivial equilibria. For the first pair, we have relations (3.10) and

$$
\begin{aligned}
y_{10}^{2} & =\frac{\left(\widehat{c}_{7}-\widehat{c}_{8}\right)^{2}-4\left[\left(b_{1}+b_{2}\right) \mu_{2}+d_{2}^{(1)}+e_{0}\right]}{16 \gamma_{1}}, \\
Y_{2} & =-\frac{1}{2}\left[\varepsilon\left(\widehat{c}_{7}-\widehat{c}_{8}\right)+\varepsilon^{2}\left[\left(b_{7}-b_{8}\right) \mu_{2}+d_{2}^{(2)}+2 \gamma_{2} y_{10}^{2}\right]\right] y_{10}+O\left(\varepsilon^{3}\right) .
\end{aligned}
$$

The second pair is described by relations (3.11), in which the expression for $y_{20}$ differs from the expression for $y_{10}$ of (4.8) in the sign in front of $e_{0}$; the equilibrium value of $Y_{1}$ is obtained from $Y_{2}$ by replacing $y_{10}$ with $y_{20}$ and by removing the minus sign in front of the main part.

These equilibria exist under the condition

$$
\gamma_{1}\left[\left(\widehat{c}_{7}-\widehat{c}_{8}\right)^{2}-4\left[\left(b_{1}+b_{2}\right) \mu_{2}+d_{2}^{(1)} \pm e_{0}\right]\right]>0,
$$

where the upper and lower signs refer to the first and second pairs.

The analysis shows that in the case $\gamma_{1}>0$ the first of the pairs of equilibria in the existence region is unstable, and for the second pair in the existence region necessary and sufficient stability conditions are fulfilled. 
If $\gamma_{1}<0$, then the second pair of equilibria is unstable. For the first pair there is a region of fulfillment only of necessary stability conditions, which is given by conditions (4.6) and the inequality

$$
\frac{1}{4}\left(\widehat{c}_{7}-\widehat{c}_{8}\right)^{2}-e_{0}<\left(b_{1}+b_{2}\right) \mu_{2}+d_{2}^{(1)}<\frac{3}{4}\left(\widehat{c}_{7}-\widehat{c}_{8}\right)^{2}-\sqrt{2 e_{0}}\left|\widehat{c}_{7}-\widehat{c}_{8}\right| .
$$

Outside this region, this pair of equilibria is unstable.

The stability pattern in the plane of parameters $x=\alpha-\alpha_{*}, y=\beta-\beta_{*}$ for a fixed value of $\varepsilon$ is shown in Fig. 2a, b for the cases $\gamma_{1}>0$ and $\gamma_{1}<0$, respectively. On the curves $g_{ \pm}$, the relations

$$
\left(b_{1}+b_{2}\right) \mu_{2}+d_{2}^{(1)}=\frac{1}{4}\left(\widehat{c}_{7}-\widehat{c}_{8}\right)^{2} \pm e_{0}
$$

are satisfied. If we assume that $d_{2}^{(1)}=s_{2} \nu_{1}^{2}+s_{1} \nu_{1}+s_{0}\left(s_{j}=\right.$ const $)$ and $\kappa_{1}=\left(\widehat{c}_{7}-\widehat{c}_{8}\right) / \nu_{1}$, then the equations of these curves are

$$
\left(a_{1}+a_{2}\right) x+\left(b_{1}+b_{2}\right) y=\left(\frac{1}{4} \kappa_{1}^{2}-s_{2}\right) x^{2}+\varepsilon s_{1} x+\varepsilon^{2}\left(s_{0} \pm e_{0}\right) .
$$

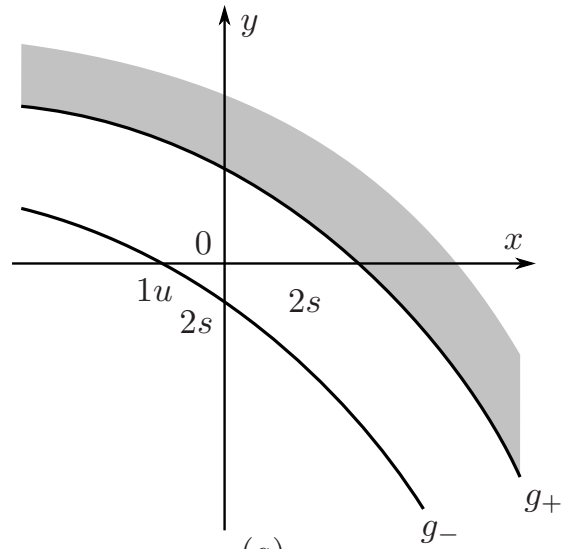

(a)

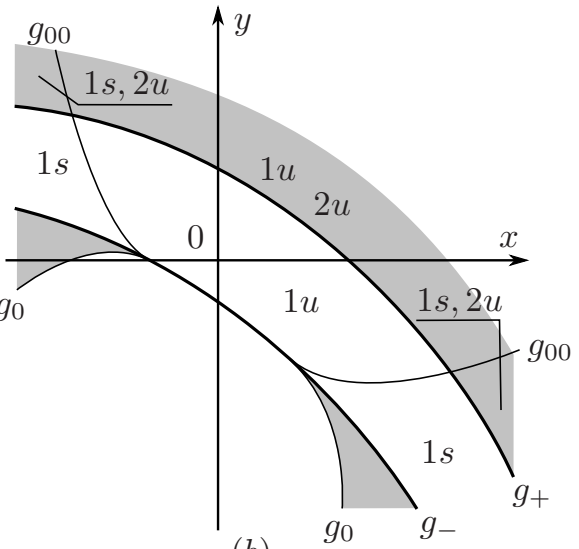

(b)

Fig. 2. The stability pattern for the second-approximation system.

On the curves $g_{0}$ and $g_{00}$, the quantity $\left(b_{1}+b_{2}\right) \mu_{2}+d_{2}^{(1)}$ takes, respectively, the values

$$
-\frac{e_{0}^{2}}{\left(\widehat{c}_{7}-\widehat{c}_{8}\right)^{2}} \text { and } \frac{3}{4}\left(\widehat{c}_{7}-\widehat{c}_{8}\right)^{2}-\sqrt{2 e_{0}}\left|\widehat{c}_{7}-\widehat{c}_{8}\right| \text {. }
$$

The common points of the curves $g_{0}, g_{00}$ and $g_{-}$correspond to the values of $\nu_{1}$ defined by the equation $\left(\widehat{c}_{7}-\widehat{c}_{8}\right)^{2}=2 e_{0}$, and the common points of the curves $g_{00}$ and $g_{+}$correspond to the values of $\nu_{1}$ defined by the equation $\left(\widehat{c}_{7}-\widehat{c}_{8}\right)^{2}=2(1+\sqrt{2})^{2} e_{0}$.

The region of stability of the trivial equilibrium of the system in Fig. 2 is colored. The numbers and letters inside the regions have the same meaning as those in Fig. 1.

When $e_{0}=0$, the equilibrium values of $y_{10}$ and $y_{20}$ (if any) lie on the circle

$$
y_{10}^{2}+y_{20}^{2}=\frac{\left(\widehat{c}_{7}-\widehat{c}_{8}\right)^{2}-4\left[\left(b_{1}+b_{2}\right) \mu_{2}+d_{2}^{(1)}\right]}{16 \gamma_{1}}
$$

and correspond to unstable equilibria of the model system. For the value of $\mu_{2}$ which lets the first expression in (4.4) vanish, this circle shrinks to a point. 


\subsection{The case $e_{0}=0$}

4.4.1. At nonzero value of the first expression in (4.4), the case $e_{0}=0$ is an analog of the case $\widetilde{d}=0$ for the model system of first approximation (see below, Section 7 ). In this case, the investigation is carried out in a neighborhood of the origin of the phase space given by the relations $y_{j}=z_{j}, Y_{j}=\varepsilon Z_{j}(j=1,2)$. This case is not considered in what follows.

4.4.2. If both quantities in (4.4) vanish, the model system of third approximation is considered which is obtained by taking account of terms of order $\varepsilon^{3}$ in the quadratic part of the Hamiltonian. The investigation is carried out in the $\varepsilon^{3 / 2}$-neighborhood of the origin of the phase space.

For the above-mentioned model system, the structure of the parts $K_{22}$ and $K_{23}$ is similar to those of the forms (4.1) and (4.2), respectively. The analysis of the system shows that, if $\widehat{c}_{7} \neq \widehat{c}_{8}$, its trivial equilibrium is stable under the condition $\left(b_{1}+b_{2}\right) \mu_{3}+d_{3}^{(1)}>0$; when the sign of this inequality is reversed, instability takes place. There are no other equilibrium points.

When $\widehat{c}_{7}=\widehat{c}_{8}$, the system has nontrivial equilibria. This condition leads to the equation $\nu_{1}=0$ and the bifurcation value $\mu_{1}=0$. In this case, it is more appropriate to assume that the value of $\nu_{1}$ is initially small (of order $\varepsilon$ ) and the neighborhood of the resonant point under study has a width of order $\varepsilon^{2}$. This case is considered in the following section.

\section{Investigation of the $\varepsilon^{2}$-neighborhood of the resonant point}

Let us come back to the initial system and consider the $\varepsilon^{2}$-neighborhood of the resonant point, which is given by the relations

$$
\alpha=\alpha_{*}+\varepsilon^{2} \nu_{2}, \quad \beta=\beta_{*}+\varepsilon^{2} \mu_{2}+\varepsilon^{3} \mu_{3}+\ldots .
$$

Normalizing the quadratic part of the Hamiltonian in terms through order three in $\varepsilon$, we obtain

$$
\begin{gathered}
\Gamma_{21}=0, \quad \Gamma_{22}=\frac{1}{2}\left(c_{1}^{\prime}+c_{2}^{\prime}+l_{1}\right)\left(y_{1}^{2}+y_{2}^{2}\right)+\frac{1}{2}\left(c_{7}^{\prime}-c_{8}^{\prime}+l_{2}\right)\left(y_{1} Y_{2}-y_{2} Y_{1}\right), \\
\Gamma_{23}=\frac{1}{2}\left[\left(b_{1}+b_{2}\right) \mu_{3}+n_{1}+n_{0}\right] y_{1}^{2}+\frac{1}{2}\left[\left(b_{1}+b_{2}\right) \mu_{3}+n_{1}-n_{0}\right] y_{2}^{2}+\frac{1}{2}\left[\left(b_{7}-b_{8}\right) \mu_{3}+n_{2}\right]\left(y_{1} Y_{2}-y_{2} Y_{1}\right) .
\end{gathered}
$$

Here the notation $c_{j}^{\prime}=a_{j} \nu_{2}+b_{j} \mu_{2}$ is introduced, and $l_{i}$ and $n_{j}$ denote constants independent of $\nu_{2}$ and $\mu_{k}$.

In considering the second-approximation system, we find that the inequality $c_{1}^{\prime}+c_{2}^{\prime}+l_{1}>0$ is a necessary and sufficient condition for the stability of its trivial equilibrium. When its sign is reversed, instability takes place. Nontrivial equilibria of the system exist under the condition $\gamma_{1}\left(c_{1}^{\prime}+c_{2}^{\prime}+l_{1}\right)<0$ and form a family of unstable singular points described by the relations

$$
\begin{gathered}
y_{1}=y_{10}+O(\varepsilon), y_{2}=y_{20}+O(\varepsilon), Y_{1}=\varepsilon^{2} Y_{12}+O\left(\varepsilon^{3}\right), Y_{2}=\varepsilon^{2} Y_{22}+O\left(\varepsilon^{3}\right) \\
y_{10}^{2}+y_{20}^{2}=-\frac{c_{1}^{\prime}+c_{2}^{\prime}+l_{1}}{4 \gamma_{1}}, Y_{12}=\left(\frac{c_{7}^{\prime}-c_{8}^{\prime}+l_{2}}{2}-\frac{\gamma_{2}\left(c_{1}^{\prime}+c_{2}^{\prime}+l_{1}\right)}{4 \gamma_{1}}\right) y_{20} \\
Y_{22}=-\left(\frac{c_{7}^{\prime}-c_{8}^{\prime}+l_{2}}{2}-\frac{\gamma_{2}\left(c_{1}^{\prime}+c_{2}^{\prime}+l_{1}\right)}{4 \gamma_{1}}\right) y_{10}
\end{gathered}
$$


The transition to a third-approximation system occurs under the condition $c_{1}^{\prime}+c_{2}^{\prime}+l_{1}=0$, which gives the bifurcation value

$$
\mu_{2}=\mu_{20}=-\frac{a_{1}+a_{2}}{b_{1}+b_{2}} \nu_{2}-\frac{l_{2}}{b_{1}+b_{2}} .
$$

We have

$$
\Gamma_{22}=\frac{1}{2}\left(\widetilde{c}_{7}-\widetilde{c}_{8}+l_{2}\right)\left(y_{1} Y_{2}-y_{2} Y_{1}\right),
$$

where the tilde means that the substitution $\mu_{2}=\mu_{20}$ into the corresponding coefficient has been made.

The trivial equilibrium of the system is stable under the condition

$$
\left(b_{1}+b_{2}\right) \mu_{3}+n_{1}-n_{0}>0 .
$$

The pair of nontrivial equilibria which exists under the condition $\gamma_{1}\left[\left(b_{1}+b_{2}\right) \mu_{3}+n_{1}+n_{0}\right]<0$ is given by relations (3.10) and by

$$
y_{10}^{2}=-\frac{\left(b_{1}+b_{2}\right) \mu_{3}+n_{1}+n_{0}}{4 \gamma_{1}}, \quad Y_{2}=-\frac{1}{2} \varepsilon^{2}\left(c_{7}^{\prime}-c_{8}^{\prime}+l_{2}\right) y_{10}+O\left(\varepsilon^{3}\right) .
$$

The second pair, which exists under the condition $\gamma_{1}\left[\left(b_{1}+b_{2}\right) \mu_{3}+n_{1}-n_{0}\right]<0$, is given by relations (3.11) and by

$$
y_{20}^{2}=-\frac{\left(b_{1}+b_{2}\right) \mu_{3}+n_{1}-n_{0}}{4 \gamma_{1}}, \quad Y_{1}=\frac{1}{2} \varepsilon^{2}\left(c_{7}^{\prime}-c_{8}^{\prime}+l_{2}\right) y_{20}+O\left(\varepsilon^{3}\right) .
$$

In the existence region, the first pair of equilibria is always unstable, and the second pair is linearly stable for $\gamma_{1}>0$ and unstable for $\gamma_{1}<0$.

These results are in qualitative agreement with the results of investigation of the firstapproximation system. We have a partitioning of the plane $x, y$ (with a fixed value of $\varepsilon$ ) into three regions whose boundaries are two parallel straight lines

$$
\left(a_{1}+a_{2}\right) x+\left(b_{1}+b_{2}\right) y+\varepsilon^{2} l_{2}+\varepsilon^{3}\left(n_{1} \pm n_{0}\right)=0,
$$

which are offset from the origin by a distance of order $\varepsilon^{2}$; the distance between the straight lines themselves is of order $\varepsilon^{3}$.

The existence regions and the stability pattern of equilibria are similar to those shown in Fig. 1.

\section{Periodic motions of complete systems}

\subsection{On periodic motions of complete systems}

Let us come back to the complete transformed nonautonomous systems with the Hamiltonians $\Gamma^{(1)}$ of $(3.5), \Gamma^{(2)}$ of (4.3) and with the Hamiltonian of third approximation from Section 5. In the previous sections it was shown that the corresponding model systems can have one or two pairs of isolated nontrivial equilibria. Below we exclude from consideration the families of equilibrium points and equilibria that correspond to the cases of multiple roots of characteristic equations.

It can be shown that for the model system of the $k$ th approximation $(k=1,2,3)$ the roots of the characteristic equations of the linearized (in the neighborhood of the above-mentioned 
equilibria) equations of perturbed motion are of order $\varepsilon^{k / 2}$ (see also Section 6.2), whereas the frequency of small time-periodic perturbation in complete nonautonomous systems is of the order of unity.

Thus, the nonresonant case of Poincaré's theory of periodic motions [18] takes place, and each nontrivial equilibrium of the model system gives rise to a unique solution, analytic in $\varepsilon$ and time-periodic with period $T$, of the complete transformed nonautonomous system. We recall that $T=4 \pi$ and $T=2 \pi$, respectively, for odd and even values of $n$ in resonant relation (see the end of Section 2). In the initial variables they correspond to motions $T$-periodic in time and analytic in fractional (for $k=1,3$ ) or integer (for $k=2$ ) powers of the small parameter.

By virtue of continuity in $\varepsilon$ of the characteristic exponents of the linearized equations of perturbed motion, the periodic motions of the complete system which are generated by linearly stable and unstable equilibria of the model systems remain, respectively, linearly stable and unstable.

\subsection{Nonlinear analysis of the stability of periodic motions}

For periodic motions of the complete linearly stable systems, a complete nonlinear analysis of stability can be carried out. For this purpose, the Hamiltonian of the complete transformed system is normalized in terms through degree four in perturbations in a neighborhood of these motions.

Taking into account the application considered in Section 8, we describe the results of nonlinear analysis of the stability of periodic motions of systems of first and third (in the $\varepsilon^{2}$-neighborhood) approximations. The analysis for the second-approximation system is similar; however, in the general form this analysis is rather cumbersome due to a large number of parameters, and is more suitable for a concrete problem.

6.2.1. Consider periodic motions generated by the equilibria (3.11) of the first-approximation system in the region of their linear stability, which is given by the conditions $\gamma_{1}>0$, $C_{1}=c_{1}+c_{2}<\tilde{d}$

The frequencies of small oscillations of the linearized equations of perturbed motion are calculated from the formulae

$$
\Omega_{1}=\sqrt{2 \widetilde{d}} \varepsilon^{1 / 2}+O\left(\varepsilon^{3 / 2}\right), \quad \Omega_{2}=\sqrt{2\left(\widetilde{d}-C_{1}\right)} \varepsilon^{1 / 2}+O\left(\varepsilon^{3 / 2}\right)
$$

After normalizing the quadratic part of the Hamiltonian of perturbed motion and normalizing the main part in the fourth-degree terms, we obtain a transformed Hamiltonian which has the following form in the new variables $z_{j}, Z_{j}(j=1,2)$ :

$$
\begin{gathered}
F=F_{20}+\varepsilon^{1 / 2} F_{40}+\varepsilon F_{41}+O\left(\varepsilon^{2}\right) \\
F_{20}=\frac{1}{2} \Omega_{1}\left(z_{1}^{2}+Z_{1}^{2}\right)+\frac{1}{2} \Omega_{2}\left(z_{2}^{2}+Z_{2}^{2}\right) \\
F_{40}=\frac{1}{4} C_{20}\left(z_{1}^{2}+Z_{1}^{2}\right)^{2}+\frac{1}{4} C_{11}\left(z_{1}^{2}+Z_{1}^{2}\right)\left(z_{2}^{2}+Z_{2}^{2}\right)+\frac{1}{4} C_{02}\left(z_{2}^{2}+Z_{2}^{2}\right)^{2} \\
C_{20}=\frac{3}{4} \frac{\gamma_{1}}{\widetilde{d}}+O(\varepsilon), C_{11}=\frac{\gamma_{1}}{\sqrt{\widetilde{d}\left(\widetilde{d}-C_{1}\right)}}+O(\varepsilon), C_{02}=\frac{3}{4} \frac{\gamma_{1}}{\left(\widetilde{d}-C_{1}\right)}+O(\varepsilon), \\
F_{41}=\frac{\sqrt{2}}{2}\left[\frac{3 \gamma_{2} C_{1}-4 C_{2} \gamma_{1}+2 \gamma_{2} \widetilde{d}}{\widetilde{d}^{1 / 2}} Z_{1} Z_{2}+\frac{4 C_{2} \gamma_{1}-\gamma_{2} C_{1}-2 \gamma_{2} c_{0}}{\left(\widetilde{d}-C_{1}\right)^{1 / 2}} z_{1} z_{2}\right] \frac{\sqrt{\widetilde{d}} z_{2}^{2}+\sqrt{\widetilde{d}-C_{1}} Z_{1}^{2}}{\left|C_{1}\right| \widetilde{d} 1 / 4\left(\widetilde{d}-C_{1}\right)^{1 / 4}} .
\end{gathered}
$$

The term $O\left(\varepsilon^{2}\right)$ in (6.1) is $T$-periodic in time. 
We first consider the nonresonant case for which the quantities $\Omega_{1}$ and $\Omega_{2}$ are not related by the fourth-order resonant relation. Then, using another canonical change of variables, the form $F_{41}$ can be eliminated.

We analyze the normalized Hamiltonian obtained above. For sufficiently small values of $\varepsilon$ the quantity

$$
\widetilde{\Delta}=C_{11}^{2}-4 C_{20} C_{02}=-\frac{5}{4} \frac{\gamma_{1}^{2}}{\widetilde{d}\left(\widetilde{d}-C_{1}\right)}+O(\varepsilon)
$$

is nonzero, and the periodic solutions under study are stable for most (in the sense of Lebesgue measure) initial conditions [17]. In addition, the quadratic form $C_{20} r_{1}^{2}+C_{11} r_{1} r_{2}+C_{02} r_{2}^{2}$ is positive definite, and formal stability [10, 19] takes place.

Consider resonant cases. In the region of existence of the periodic solutions under study, for the values $C_{1}=(8 / 9) \widetilde{d}+O(\varepsilon)$ and $C_{1}=-8 \widetilde{d}+O(\varepsilon)$ we have fourth-order resonances $\Omega_{1}=3 \Omega_{2}$ and $3 \Omega_{1}=\Omega_{2}$, respectively. In these cases, the form $F_{41}$ cannot be completely eliminated: there remain resonant terms in it.

We continue the normalization of the Hamiltonian in the terms of degrees six, eight etc. in perturbations, taking into account the existence of resonances of orders eight, twelve etc. Since the eigenfrequencies $\Omega_{j}$ (of order $\varepsilon^{1 / 2}$ ) are incommensurable with the frequency of external perturbation (of the order of unity), time $t$ can be eliminated at each step of normalization. As a result, we have an autonomous Hamiltonian which is a formal first integral of the system, whose main part, of order $\varepsilon^{1 / 2}$, is positive definite (and the resonant terms are of order $\varepsilon$ and higher). In a sufficiently small neighborhood of the origin, this formal integral is also positive definite. Therefore, in resonant cases the periodic motions of interest are formally stable.

6.2.2. For the third-approximation system from Section 5, we consider periodic motions generated by the equilibria (3.11) and (5.3) in the linear stability region $\gamma_{1}>0$, $B=\left(b_{1}+b_{2}\right) \mu_{3}+n_{1}<n_{0}$.

The frequencies of small oscillations of the linearized equations of perturbed motion are calculated from the formulae

$$
\Omega_{1}=\sqrt{2 n_{0}} \varepsilon^{3 / 2}+O\left(\varepsilon^{5 / 2}\right), \quad \Omega_{2}=\sqrt{2\left(n_{0}-B\right)} \varepsilon^{3 / 2}+O\left(\varepsilon^{5 / 2}\right),
$$

and the normalized Hamiltonian of perturbed motion has the form

$$
F=F_{20}+\varepsilon^{3 / 2} F_{40}+\varepsilon^{2} F_{41}+O\left(\varepsilon^{3}\right),
$$

in which the parts $F_{20}$ and $F_{40}$ are calculated from the formulae (6.2) and (6.3) and

$$
\begin{aligned}
C_{20} & =\frac{3}{4} \frac{\gamma_{1}}{n_{0}}+O(\varepsilon), \quad C_{11}=\frac{\gamma_{1}}{\sqrt{n_{0}\left(n_{0}-B\right)}}+O(\varepsilon), \quad C_{02}=\frac{3}{4} \frac{\gamma_{1}}{\left(n_{0}-B\right)}+O(\varepsilon), \\
F_{41} & =\frac{\sqrt{2}\left(\widetilde{c}_{7}-\widetilde{c}_{8}+l_{2}\right) \gamma_{1}\left(z_{1} z_{2} \sqrt{n_{0}}-Z_{1} Z_{2} \sqrt{n_{0}-B}\right)\left(\sqrt{n_{0}} z_{2}^{2}+\sqrt{n_{0}-B} Z_{1}^{2}\right)}{|B| n_{0}^{3 / 4}\left(n_{0}-B\right)^{3 / 4}} .
\end{aligned}
$$

Analyzing the expressions for $C_{i j}$ and the quantity $\widetilde{\Delta}$, which was introduced in (6.4), we conclude that the periodic solutions under study are stable for most initial conditions and are formally stable.

In the region of existence of periodic motions, for the values $B=(8 / 9) n_{0}+O(\varepsilon)$ and $B=-8 n_{0}+O(\varepsilon)$ we have fourth-order resonances $\Omega_{1}=3 \Omega_{2}$ and $3 \Omega_{1}=\Omega_{2}$, respectively. Following the same reasoning as above, we conclude that in these resonant cases the periodic motions are formally stable. 


\section{The first-approximation system: the case $\widetilde{d}=0$}

\subsection{Further transformations of the model Hamiltonian}

Let us return to the analysis of the first-approximation system, assuming that the quantity $\tilde{d}$ introduced in Section 3.1 vanishes. In this case, the form $\Gamma_{21}$ reads

$$
\Gamma_{21}=\frac{1}{2} C_{1}\left(y_{1}^{2}+y_{2}^{2}\right)+\frac{1}{2} C_{2}\left(y_{1} Y_{2}-y_{2} Y_{1}\right), \quad C_{1}=c_{1}+c_{2}, \quad C_{2}=c_{7}-c_{8} .
$$

In what follows, we will assume that $C_{1} \neq 0$.

In the complete system with the Hamiltonian $\Gamma^{(1)}$ of $(3.5)$, we perform some additional canonical transformations. We first make the change of variables

$$
y_{j}=z_{j}, \quad Y_{j}=\varepsilon^{1 / 2} Z_{j} \quad(j=1,2) .
$$

As a result, this Hamiltonian becomes

$$
S=\varepsilon^{1 / 2}\left[\frac{1}{2}\left(Z_{1}^{2}+Z_{2}^{2}\right)+\frac{1}{2} C_{1}\left(z_{1}^{2}+z_{2}^{2}\right)+\gamma_{1}\left(z_{1}^{2}+z_{2}^{2}\right)^{2}\right]+O(\varepsilon) .
$$

Here, as above, the term $O(\varepsilon)$ is $T$-periodic in $t$.

Next, we make the transformation $z_{j}, Z_{j} \rightarrow u_{j}, U_{j}(j=1,2)$

$$
\begin{aligned}
z_{1} & =u_{1} \cos \left(\frac{n}{2} t\right)+u_{2} \sin \left(\frac{n}{2} t\right), & z_{2} & =-u_{1} \sin \left(\frac{n}{2} t\right)+u_{2} \cos \left(\frac{n}{2} t\right), \\
Z_{1} & =U_{1} \cos \left(\frac{n}{2} t\right)+U_{2} \sin \left(\frac{n}{2} t\right), & Z_{2} & =-U_{1} \sin \left(\frac{n}{2} t\right)+U_{2} \cos \left(\frac{n}{2} t\right),
\end{aligned}
$$

which is similar to the transformation inverse to (2.5).

This gives the Hamiltonian

$$
\widehat{S}=\frac{n}{2}\left(u_{1} U_{2}-u_{2} U_{1}\right)+\varepsilon^{1 / 2}\left[\frac{1}{2}\left(U_{1}^{2}+U_{2}^{2}\right)+\frac{1}{2} C_{1}\left(u_{1}^{2}+u_{2}^{2}\right)+\gamma_{1}\left(u_{1}^{2}+u_{2}^{2}\right)^{2}\right]+O(\varepsilon) .
$$

We now transform to polar coordinates by making the canonical transformation $u_{1}, u_{2}, U_{1}, U_{2} \rightarrow r, \theta, R, J$ by the formulae

$$
\begin{aligned}
u_{1}=r \cos \theta, & u_{2}=r \sin \theta \\
U_{1}=R \cos \theta-\frac{J}{r} \sin \theta, & U_{2}=R \sin \theta+\frac{J}{r} \cos \theta .
\end{aligned}
$$

The transformed Hamiltonian becomes

$$
\widetilde{S}=\frac{n}{2} J+\varepsilon^{1 / 2}\left[\frac{1}{2}\left(R^{2}+\frac{J^{2}}{r^{2}}\right)+\frac{1}{2} C_{1} r^{2}+\gamma_{1} r^{4}\right]+O(\varepsilon) .
$$

Finally, we set

$$
r=\left|\gamma_{1}\right|^{-1 / 2} \varrho, \quad R=\left|\gamma_{1}\right|^{-1 / 2} P, \quad \theta=\varphi, \quad J=\left|\gamma_{1}\right|^{-1} I .
$$

The complete transformed Hamiltonian takes the form

$$
\begin{gathered}
F=F^{\prime}+O(\varepsilon), \\
F^{\prime}=F_{0}+\varepsilon^{1 / 2} F_{1}, \quad F_{0}=\frac{n}{2} I, \quad F_{1}=\frac{1}{2}\left(P^{2}+\frac{I^{2}}{\varrho^{2}}\right)+\frac{1}{2} C_{1} \varrho^{2}+\sigma_{1} \varrho^{4} .
\end{gathered}
$$

Here the notation $\sigma_{1}=\operatorname{sign} \gamma_{1}$ is introduced and the term $O(\varepsilon)$ is $T$-periodic in time. 
We note that the Hamiltonian $F^{\prime}$ of $(7.6)$ coincides, up to notation, with the corresponding approximate Hamiltonian describing the motions of the autonomous Hamiltonian system with two degrees of freedom in the case of identical frequencies under consideration. A detailed analysis of the nonlinear oscillations of such an autonomous system in the case $\sigma_{1}=1$ is carried out in [15].

We describe the properties, necessary for further analysis, of the approximate system with the Hamiltonian (7.6) for both cases $\sigma_{1}=1$ and $\sigma_{1}=-1$. In this system, the coordinate $\varphi$ is cyclic and there is a first integral $I=c=$ const. Without loss of generality we assume that $c \geqslant 0$.

\subsection{A reduced system with one degree of freedom}

Consider a reduced system with one degree of freedom with the Hamiltonian $F_{1}$ in which the substitution $I=c$ has been made. The system has the first integral

$$
\frac{1}{2}\left(P^{2}+\frac{c^{2}}{\varrho^{2}}\right)+\frac{1}{2} C_{1} \varrho^{2}+\sigma_{1} \varrho^{4}=h=\text { const. }
$$

The phase portraits of the reduced system for $c>0$ are shown in Fig. 3 for the cases $\sigma_{1}=1$ and all $C_{1} \neq 0$ (a); $\sigma_{1}=-1$ and $C_{1}<3(2 c)^{2 / 3}$ (b); $\sigma_{1}=-1$ and $C_{1}>3(2 c)^{2 / 3}$ (c). At the equilibrium points of the system we have $P=0$, and the value $\varrho=\varrho_{0}$ is the root of the equation

$$
4 \sigma_{1} \varrho^{6}+C_{1} \varrho^{4}-c^{2}=0
$$

The solutions of the reduced system (except for solutions corresponding to the asymptotic motion in Fig. 3c) are written in terms of Jacobi elliptic functions. We restrict our attention to the closed trajectories shown in Fig. 3a, c, which correspond to oscillations of the system in a neighborhood of stable equilibrium points. Let $u_{1}, u_{2}, u_{3}\left(u_{1}>u_{2}>u_{3}\right)$ be the real roots of the equation

$$
-2 \sigma_{1} u^{3}-C_{1} u^{2}+2 h u-c^{2}=0 .
$$

The dependence $\varrho(t)$ for the oscillations in Fig. 3a is described by the relation

$$
\varrho^{2}(t)=u_{1}-\left(u_{1}-u_{2}\right) \operatorname{sn}^{2}\left(\sqrt{2\left(u_{1}-u_{3}\right)} t, k_{1}\right), \quad k_{1}^{2}=\frac{u_{1}-u_{2}}{u_{1}-u_{3}}
$$

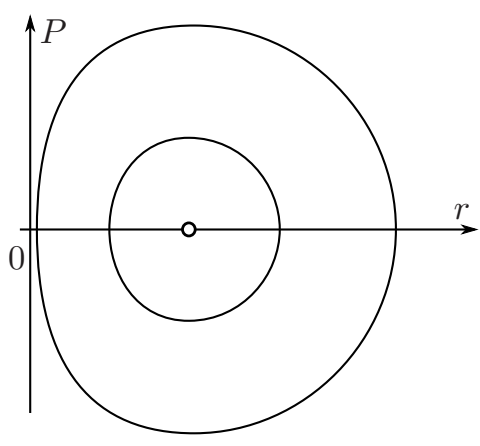

(a)

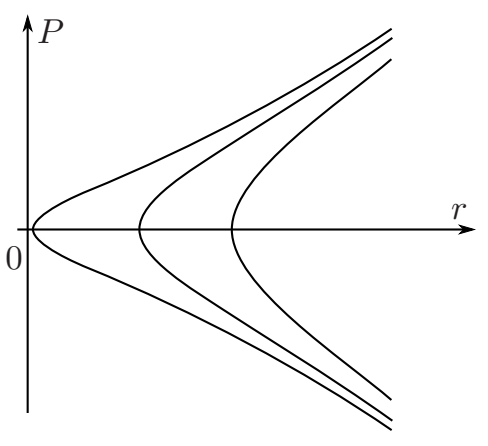

(b)

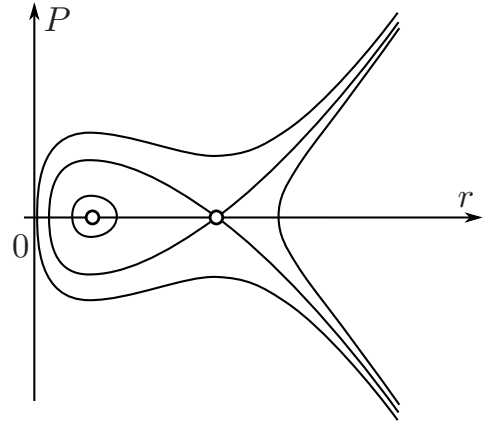

(c)

Fig. 3. Phase portraits of the reduced system for $c>0$. 
where $0<u_{2} \leqslant \varrho^{2} \leqslant u_{1}$ and it is assumed that $\varrho^{2}(0)=u_{1}$. For the oscillations in Fig. 3c we have

$$
\varrho^{2}(t)=u_{3}+\left(u_{2}-u_{3}\right) \operatorname{sn}^{2}\left(\sqrt{2\left(u_{1}-u_{3}\right)} t, k_{2}\right), \quad k_{2}^{2}=\frac{u_{2}-u_{3}}{u_{1}-u_{3}},
$$

where $0<u_{3} \leqslant \varrho^{2} \leqslant u_{2}$ and $\varrho^{2}(0)=u_{3}$.

The frequencies of the oscillations described above are calculated from the formulae

$$
\omega_{1,2}=\frac{\pi \sqrt{2\left(u_{1}-u_{3}\right)}}{K\left(k_{1,2}\right)}
$$

where $K\left(k_{1,2}\right)$ is the complete elliptic integral of the first kind.

After finding the dependence $\varrho(t)$ the corresponding dependence $P(t)$ is defined using the integral (7.7).

For $c=0$ the phase portraits of the reduced system have the form shown in Figs. $4 \mathrm{a}-4 \mathrm{~d}$ for the cases $\sigma_{1}=1, C_{1}>0$ (a), $\sigma_{1}=1, C_{1}<0$ (b), $\sigma_{1}=-1, C_{1}>0$ (c), $\sigma_{1}=-1, C_{1}<0$ (d). In these figures, it is formally assumed that the variable $r$ can take values of any sign. The phase trajectories are symmetric relative to the ordinate axis, and the symmetric parts of the trajectories from the right and left half-planes correspond to the same motion of the system.

The solutions corresponding to oscillations in the case $c=0$ are described by the same formulae as in the general case $c>0$. For $\sigma_{1}=1$ and $h>0$, for trajectories corresponding to oscillations in a neighborhood of trivial equilibrium (Fig. 4a) and the closed trajectories enclosing the separatrix in Fig. $4 \mathrm{~b}$, we use the formula (7.9), where $u_{1}=\varrho_{1}^{2}, u_{2}=0, u_{3}=\varrho_{2}^{2}$.

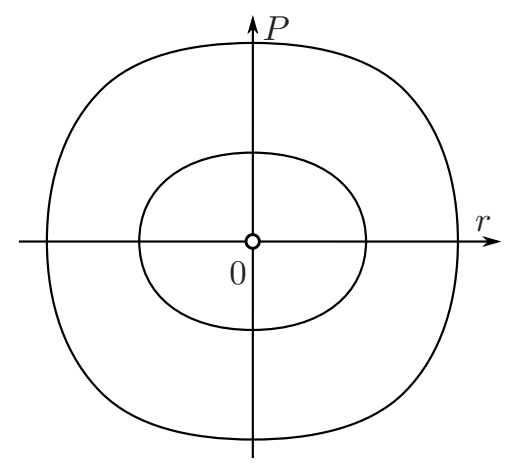

(a)

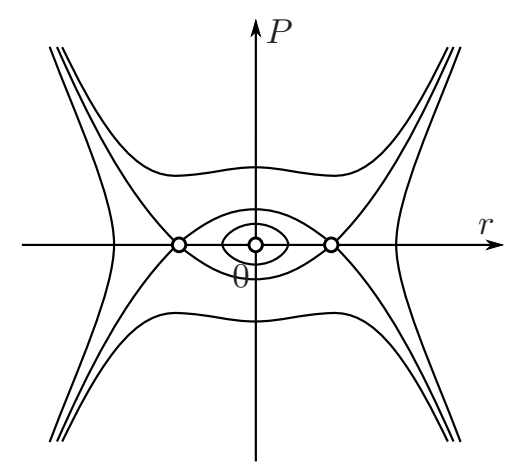

(c)

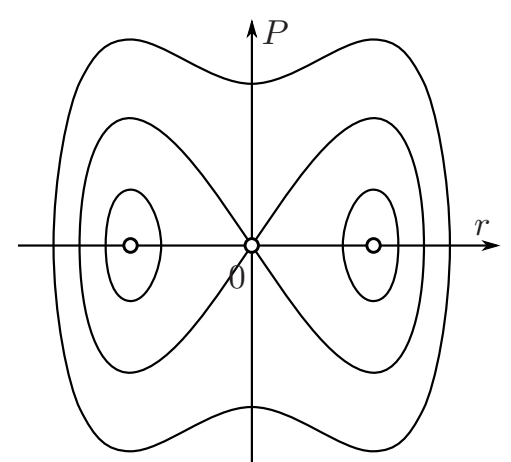

(b)

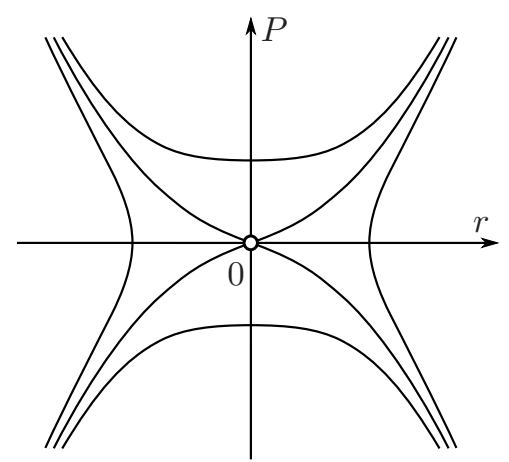

(d)

Fig. 4. Phase portraits of the reduced system for $c=0$. 
For $\sigma_{1}=1$ and $-C_{1}^{2} / 16<h<0$, for oscillations in the neighborhood of stable equilibria in Fig. 4b we take the same formula, in which we assume $u_{1}=\varrho_{1}^{2}, u_{2}=\varrho_{2}^{2}, u_{3}=0$. Here the notation $\varrho_{1,2}^{2}=\left(-C_{1} \pm \sqrt{C_{1}^{2}+16 h} / 4\left(\varrho_{1}>\varrho_{2}\right)\right.$ is introduced.

In the case $\sigma_{1}=-1$ and $0<h<C_{1}^{2} / 16$, for oscillations in the neighborhood of trivial equilibrium in Fig. 4c we use the formula (7.10), where $u_{1}=\varrho_{1}^{2}, u_{2}=\varrho_{2}^{2}, u_{3}=0$, assuming that $\varrho_{1,2}^{2}=\left(C_{1} \pm \sqrt{C_{1}^{2}-16 h} / 4\left(\varrho_{1}>\varrho_{2}\right)\right.$.

The frequencies of the above oscillations are calculated from the formulae (7.11) for the chosen values of $u_{j}$.

\subsection{Verification of the nondegeneracy condition in the regions of oscillations of the reduced system}

In the ranges of parameters corresponding to oscillations of the reduced system with one degree of freedom, we introduce action-angle variables $I, w_{1}$ [17], assuming that

$$
I_{1}=I_{1}(h, c)=\frac{1}{2 \pi} \oint P d \varrho .
$$

Here integration is performed along the closed trajectories under consideration. The quantity $P=P(\varrho, h, c)$ in the integrand is defined from relation (7.7). We have

$$
I_{1}(h, c)=\frac{1}{2 \pi} \int \frac{\sqrt{-2 \sigma_{1} \varrho^{6}-C_{1} \varrho^{4}+2 h \varrho^{2}-c^{2}}}{\varrho} d \varrho .
$$

The upper and lower bounds of integration correspond to the boundaries of the variation interval of $\varrho$ for the trajectory under study.

The function $h=h\left(I_{1}, c\right)$ inverse to (7.12) is the Hamiltonian of the reduced system, which is written in the action-angle variables. The frequency of the corresponding oscillation is equal to $\omega=\partial h / \partial I_{1}$.

For further analysis we need to verify the nondegeneracy condition

$$
\frac{\partial^{2} h}{\partial I_{1}^{2}} \neq 0
$$

of the Hamiltonian $h=h\left(I_{1}, c\right)$. Taking into account that

$$
\frac{\partial^{2} h}{\partial I_{1}^{2}}=\frac{\partial \omega}{\partial I_{1}}=\omega \frac{\partial \omega}{\partial h}
$$

and using the formulae (7.8)-(7.11) and some relations from the theory of Jacobi elliptic integrals, we find that on the trajectories corresponding to oscillations in the neighborhood of stable equilibria in Fig. 3a, c the left-hand sides of (7.13) are, respectively, equal to

$$
\frac{\partial^{2} h}{\partial I_{1}^{2}}=-\frac{\pi^{2}\left[2 k_{2}^{2} K\left(k_{1}\right)\left(u_{1}-u_{2}\right)\left(3 u_{1} u_{3}+h\right)+\left(E\left(k_{1}\right)-k_{2}^{2} K\left(k_{1}\right)\right)\left(9 c^{2}+C_{1} h\right)\right]}{2 K^{3}\left(k_{1}\right)\left(u_{1}-u_{2}\right)^{2}\left(u_{2}-u_{3}\right)^{2}}
$$

and

$$
\frac{\partial^{2} h}{\partial I_{1}^{2}}=\frac{\pi^{2}\left[2 k_{1}^{2} K\left(k_{2}\right)\left(u_{2}-u_{3}\right)\left(-h+3 u_{1} u_{3}\right)-\left(E\left(k_{2}\right)-k_{1}^{2} K\left(k_{2}\right)\right)\left(-9 c^{2}+C_{1} h\right)\right]}{2 K^{3}\left(k_{2}\right)\left(u_{1}-u_{2}\right)^{2}\left(u_{2}-u_{3}\right)^{2}} .
$$

We note that the first of these formulae was obtained previously in [15], but there it contained some inaccuracies; in (7.14) these inaccuracies were removed. 
Numerical calculations have shown that in the case $\sigma_{1}=1$ the numerator in the formula (7.14) vanishes on some two-dimensional surface in the space of parameters $c, C_{1}, h$ when $C_{1}<0$ and, thus, on this surface, the nondegeneracy condition is violated. In the other part of the parameter space in the case $\sigma_{1}=1$, and for all admissible parameter values in the case $\sigma_{1}=-1$, the nondegeneracy condition is fulfilled.

In the case $c=0$, for the three oscillation patterns described at the end of Section 7.2, we have, respectively,

$$
\begin{aligned}
& \frac{\partial^{2} h}{\partial I_{1}^{2}}=\frac{\pi^{2}\left[-2 C_{1} E\left(k_{1}\right)+K\left(k_{1}\right)\left(C_{1}+\sqrt{C_{1}^{2}+16 h}\right)\right]}{4 K^{3}\left(k_{1}\right) h} \\
& \frac{\partial^{2} h}{\partial I_{1}^{2}}=\frac{2 \pi^{2}\left(C_{1}-\sqrt{\left.C_{1}^{2}+16 h\right)\left[C_{1} E\left(k_{1}\right)-K\left(k_{1}\right)\left(C_{1}+\sqrt{C_{1}^{2}+16 h}\right)\right]}\right.}{K^{3}\left(k_{1}\right)\left(C_{1}^{2}+16 h\right)\left(C_{1}+\sqrt{C_{1}^{2}+16 h}\right)}, \\
& \frac{\partial^{2} h}{\partial I_{1}^{2}}=-\frac{2 \pi^{2}\left(C_{1}+\sqrt{C_{1}^{2}-16 h}\right)\left[C_{1} E\left(k_{2}\right)-\sqrt{C_{1}^{2}-16 h} K\left(k_{2}\right)\right]}{K^{3}\left(k_{2}\right)\left(C_{1}^{2}-16 h\right)\left(C_{1}-\sqrt{C_{1}^{2}-16 h}\right)}
\end{aligned}
$$

where $k_{1}$ and $k_{2}$ are calculated by the formulae from (7.9) and (7.10) for the values of $u_{j}$ described at the end of this section. It is easy to verify that for all three cases the nondegeneracy condition is satisfied.

\subsection{On the motions of the complete system with the Hamiltonian (7.5), (7.6)}

7.4.1. On the motions of the model system. Let us come back to the model system with two degrees of freedom with the Hamiltonian $F^{\prime}$ of (7.6). Let $\varrho(t), R(t)$ be the solutions of the reduced system as described in Section 7.2.

The trivial equilibrium of the reduced system in Fig. 4 (the case $c=0$ ) corresponds to the trivial equilibrium of the model system (and the initial nonautonomous system). The nontrivial equilibria in Fig. $4 \mathrm{~b}$ correspond to the equilibrium point $\varrho=-C_{1} / 4, P=0, I=0$ of the system with the Hamiltonian $F^{\prime}$. Note that in the model system with the Hamiltonian $\Gamma^{(10)}$ of $(3.5)$ (with $\widetilde{d}=0$ ) this equilibrium corresponds to the family of equilibria (3.13).

The trajectories corresponding to oscillations, as shown in Fig. 4, correspond to periodic solutions (with frequency $\varepsilon^{1 / 2} \omega_{j}$ ) of the model system

$$
\varrho=\varrho\left(\varepsilon^{1 / 2} t\right), \quad R=R\left(\varepsilon^{1 / 2} t\right), \quad I=0 .
$$

In the case $c>0$, the equilibria of the reduced system in Fig. 3a, c correspond to a oneparameter family (with parameter $c$ ) of solutions of the model system

$$
\varrho=\varrho_{0}, \quad P=0, \quad I=c, \quad \varphi=\Omega_{0} t, \quad \Omega_{0}=\frac{n}{2}+\varepsilon^{1 / 2} \frac{c}{\varrho_{0}^{2}} .
$$

The trajectories corresponding to oscillations in the neighborhood of stable equilibria, as shown in Fig. 3a, c, correspond to a one-parameter family of two-frequency conditionally periodic solutions

$$
\varrho=\varrho\left(\varepsilon^{1 / 2} t\right), \quad R=R\left(\varepsilon^{1 / 2} t\right), \quad I=c, \quad \Omega=\frac{n}{2}+\varepsilon^{1 / 2} \frac{c}{\varrho^{2}\left(\varepsilon^{1 / 2} t\right)}
$$

with frequencies $\varepsilon^{1 / 2} \omega_{j}(j=1$ or 2 , see $(7.11))$ and $\Omega$.

7.4.2. On the motions of the complete system. Let us establish correspondence between the motions of the model system and the motions of the complete system with the Hamiltonian (7.5), (7.6). We restrict us to the case $c>0$. 
It obviously follows from relations (7.5) and (7.6) that the condition

$$
\frac{\partial^{2} F}{\partial I^{2}} \neq 0
$$

holds. Therefore, according to [17, 22, 23], most steady rotations (7.16) of the model system give rise to two-frequency periodic motions of the complete system with frequencies $\Omega_{0}$ from (7.16) and $2 \pi / T$.

For the parameter values corresponding to oscillations of the reduced system, we rewrite the Hamiltonian of the model system and then the Hamiltonian (7.5), (7.6) of the complete system in the variables $I_{1}, I, w_{1}, \varphi$. We obtain

$$
\Phi\left(I_{1}, I, w_{1}, \varphi, t\right)=\frac{n}{2} I+\varepsilon^{1 / 2} h\left(I_{1}, I\right)+\varepsilon \widetilde{\Phi}\left(I_{1}, I, w_{1}, \varphi, t\right),
$$

where the last term is $2 \pi$-periodic in the angle variables $w_{1}$ and $\varphi$ and $T$-periodic in $t$.

In the Hamiltonian (7.18) the case of eigendegeneracy [17] takes place since the main part (of the order of unity) of the Hamiltonian depends only on one action variable.

Consider the nondegeneracy conditions [17]

$$
\frac{\partial \Phi}{\partial I} \neq 0, \quad \frac{\partial \Phi}{\partial I_{1}} \neq 0, \quad \frac{\partial^{2} \Phi}{\partial I_{1}^{2}} \neq 0 .
$$

The first of these inequalities obviously holds by virtue of (7.18). The second condition holds since the frequencies $\omega_{1,2}$, which are calculated by the formulae (7.11), are nonzero. Finally, the third condition is equivalent to the condition for nondegeneracy of the reduced Hamiltonian which was verified in Section 7.3 and holds for all admissible values in the three-dimensional parameter space, except for points of some two-dimensional surface in the case $\sigma_{1}=1$.

This implies [17] that most two-frequency motions (7.17) of the model system in the complete system give rise to three-frequency conditionally periodic motions with frequencies $\Omega+O(\varepsilon)$, $\varepsilon \omega_{j}+O(\varepsilon)$ and $2 \pi / T$. The portion of the phase space which is not filled with such trajectories is exponentially small and is defined by the expression $O\left(-\delta^{\prime} \varepsilon^{-1 / 2}\right)$, where $\delta^{\prime}=$ const $>0$.

\section{Resonant motions of a symmetric satellite in a neighborhood of cylindrical precession}

As an application, we consider the problem of the motion of a dynamically symmetric satellite (rigid body) in a central Newtonian gravitational field in an elliptic orbit. The orientation of the principal central axes of inertia of the satellite in the orbital coordinate system $O X Y Z$ (whose axes $O X, O Y, O Z$ are directed, respectively, along the transversal to the orbit, along the binormal to the orbit's plane and along the radius vector of the center of mass relative to the attracting center) will be specified by Euler angles $\psi, \theta, \varphi$.

The motion of the symmetry axis of the satellite is described by a reduced system with two degrees of freedom with the Hamiltonian [20]

$$
\begin{aligned}
H & =\frac{p_{\psi}^{2}}{2(1+e \cos \nu)^{2} \sin ^{2} \theta}+\frac{p_{\theta}^{2}}{2(1+e \cos \nu)^{2}}-p_{\psi} \cot \theta \cos \psi-\frac{\alpha \beta\left(1-e^{2}\right)^{3 / 2} p_{\psi} \cos \theta}{(1+e \cos \nu)^{2} \sin ^{2} \theta}- \\
& -p_{\theta} \sin \psi+\frac{\alpha \beta\left(1-e^{2}\right)^{3 / 2} \cos \psi}{\sin \theta}+\frac{\alpha^{2} \beta^{2}\left(1-e^{2}\right)^{3} \cot ^{2} \theta}{2(1+e \cos \nu)^{2}}+\frac{3}{2}(\alpha-1)(1+e \cos \nu) \cos ^{2} \theta .
\end{aligned}
$$


Here, $p_{\psi}$ and $p_{\theta}$ are dimensionless momenta conjugate to the angles $\psi$ and $\theta$, and $\nu$ is the true anomaly of the orbit of the center of mass, which is taken to be an independent variable.

The Hamiltonian depends on three parameters: the eccentricity of the orbit $e$, which we assume to be small $(0<e \ll 1)$ in what follows, the inertial parameter $\alpha=C / A$, where $A$ and $C$ are the equatorial and axial moments of inertia of the satellite $(0 \leqslant \alpha \leqslant 2)$, and $\beta=r_{0} / \omega_{0}$, where $r$ is the projection of the angular velocity vector of the body in the orbital coordinate system onto the axis of dynamical symmetry $\left(r=r_{0}=\right.$ const), and $\omega_{0}$ is the average motion of the satellite's center of mass along the orbit.

There exists [21] a partial solution to the reduced system

$$
\psi=\pi, \quad \theta=\frac{\pi}{2}, \quad p_{\psi}=0, \quad p_{\theta}=0
$$

which corresponds to the steady rotation of the satellite with angular velocity $r_{0}$ about the axis of dynamical symmetry perpendicular to the plane of the orbit (cylindrical precession).

We will investigate the motions of the satellite's axis in the neighborhood of this partial solution. Let us introduce perturbations by the formulae

$$
\psi=\pi+q_{2}, \quad \theta=\frac{\pi}{2}+q_{1}, \quad p_{\psi}=p_{2}, \quad p_{\theta}=p_{1}
$$

and represent the Hamiltonian of perturbed motion in the form (2.1), in which $\varepsilon=e$ and $t=\nu$.

The equation for the frequencies of small linear oscillations of the system in the case of a circular $(e=0)$ orbit of the center of mass of the satellite has the form [21]

$$
\omega^{4}-\left(3 \alpha-1+\alpha^{2} \beta^{2}-2 \alpha \beta\right) \omega^{2}+(\alpha \beta-1)(\alpha \beta+3 \alpha-4)=0 .
$$

From this equation we find two points from the admissible part of the parameter plane $(\alpha, \beta)$ at which the resonant cases considered in this paper take place:

$$
\begin{aligned}
& \text { Point 1: } \quad \alpha=0.829862, \quad \beta=1.082050, \quad \omega_{1}=\omega_{2}=\frac{1}{2} \\
& \text { Point 2: } \quad \alpha=0.826677, \quad \beta=-1.215694, \quad \omega_{1}=\omega_{2}=\frac{3}{2} .
\end{aligned}
$$

Points 1 and 2 lie on the boundary where only necessary conditions for stability of the cylindrical precession of the symmetric satellite in the circular orbit are satisfied. For both points the normalized quadratic part of the Hamiltonian does not reduce to the sum of squares.

We note that there is also a point $\alpha=1, \beta=2$ at which equation (8.1) has a double root $\omega_{1}=\omega_{2}=1$ and the above-mentioned quadratic form reduces to the sum of squares. This case was previously examined in detail in [6].

Based on the results from the previous sections, we find resonant periodic motions of the symmetry axis of the satellite near its cylindrical precession for the parameter values from small neighborhoods of resonant points 1 and 2 in the three-dimensional parameter space.

\subsection{Point 1}

Consider the $e$-neighborhood of point 1 of the form (2.3) for $\varepsilon=e$ and resonant values of $\alpha_{*}$ and $\beta_{*}$ from (8.2). Making the univalent canonical linear change of variables

$$
\begin{aligned}
& q_{1}=0.352645 p_{1}^{\prime}-0.839103 q_{2}^{\prime}, \quad q_{2}=-1.313363 q_{1}^{\prime}+0.551959 p_{2}^{\prime}, \\
& p_{1}=0.893811 q_{1}^{\prime}-1.567384 p_{2}^{\prime}, \quad p_{2}=-1.001397 p_{1}^{\prime}+0.571053 q_{2}^{\prime},
\end{aligned}
$$


we reduce the autonomous quadratic part of the Hamiltonian to the form

$$
H_{20}=\frac{1}{2}\left(p_{1}^{\prime 2}+p_{2}^{\prime 2}\right)+\frac{1}{2}\left(q_{1}^{\prime} p_{2}^{\prime}-p_{1}^{\prime} q_{2}^{\prime}\right)
$$

The subsequent nonlinear change of variables transforms $H_{40}$ to the form (2.4), where

$$
\gamma_{1}=0.0887855, \quad \gamma_{2}=-0.299151, \quad \gamma_{3}=0.50129344 \text {. }
$$

Next, we make a transformation similar to (2.5) for $n=1$ and normalize the nonautonomous quadratic part of the Hamiltonian in the terms of order $e$. As a result, we obtain the form (3.1), where

$$
\begin{gathered}
c_{1}+c_{2}=C_{1}=0.550601 \mu_{1}+1.774066 \nu_{1}, \quad c_{0}=-0.292965, \quad d_{0}=0, \\
c_{7}-c_{8}=C_{2}=-0.768972 \nu_{1}-1.270572 \mu_{1} .
\end{gathered}
$$

We set up a corresponding model system of first approximation. Exploring its properties, we find that the trivial equilibrium of the linear system is stable under the condition

$$
0.550601 \mu_{1}+1.774066 \nu_{1}-0.292965>0
$$

and is unstable when the sign of the inequality is reversed.

Nontrivial equilibria of the nonlinear model system are described by the relations

$$
\begin{gathered}
y_{2}=0, \quad Y_{1}=0, \quad y_{1}=y_{10}+e y_{11}+O\left(e^{2}\right), \quad Y_{2}=e Y_{21}+O\left(e^{2}\right), \\
y_{10}^{2}=-1.550367 \mu_{1}-4.995368 \nu_{1}+0.824924, \\
y_{11}=\left(2.165044 \mu_{1}^{2}+9.090629 \nu_{1} \mu_{1}+5.775834 \nu_{1}^{2}-1.617792 \mu_{1}-5.738694 \nu_{1}+0.879997\right) y_{10}^{-1}, \\
Y_{21}=-\left(1.109882 \nu_{1}-0.171493 \mu_{1}-0.246777\right) y_{10}
\end{gathered}
$$

and

$$
\begin{gathered}
y_{1}=0, \quad Y_{2}=0, \quad y_{2}=y_{20}+e y_{21}+O\left(e^{2}\right), \quad Y_{1}=e Y_{11}+O\left(e^{2}\right), \\
y_{20}^{2}=-1.550367 \mu_{1}-4.995368 \nu_{1}-0.824924, \\
y_{21}=\left(2.165044 \mu_{1}^{2}+9.090629 \nu_{1} \mu_{1}+5.775834 \nu_{1}^{2}+1.617792 \mu_{1}+5.738694 \nu_{1}+0.879997\right) y_{20}^{-1}, \\
Y_{11}=\left(1.109882 \nu_{1}-0.171493 \mu_{1}+0.246777\right) y_{20} .
\end{gathered}
$$

These equilibria exist if the right-hand sides of the expressions with squares are positive. The equilibria from the first pair are linearly stable and those from the second pair are unstable in the existence regions. The stability pattern is illustrated in Fig. 1a.

The equilibria described above correspond to solutions, $4 \pi$-periodic in $\nu$ and analytic in $e$, of the complete nonautonomous transformed system. In the initial system these solutions are $4 \pi$-periodic in $\nu$ and analytic in $e^{1 / 2}$. Note that periodic solutions generated by pairs of equilibria arise from each other by shifting the independent variable $\nu$ by $2 \pi$ and hence physically correspond to the same periodic motion of the symmetry axis of the satellite.

We present the explicit form of the periodic solution to the initial system, which corresponds to the first pair of equilibria:

$$
\begin{aligned}
q_{1} & =e^{1 / 2}\left[-\left(0.172292 \mu_{1}-0.413139 \nu_{1}+0.495985\right) \sin \frac{\nu}{2}+\right. \\
& \left.+\left(0.104100 \mu_{1}+0.335416 \nu_{1}+0.174681\right) \sin \frac{3}{2} \nu\right] y_{10}+
\end{aligned}
$$




$$
\begin{aligned}
& +e^{3 / 2}\left[\left[\left(0.0420513 \mu_{1}-1.921111 \nu_{1}-1.059478\right) y_{10}+\right.\right. \\
& +\left(0.104375 \mu_{1}+1.304577 \nu_{1}-0.643195\right) y_{11}- \\
& \left.-\left(0.554885 \mu_{1}+2.501462 \nu_{1}+0.159989\right) Y_{21}\right] \sin \frac{\nu}{2}+ \\
& +\left[\left(-0.262580 \mu_{1}-0.846047 \nu_{1}-0.389228\right) y_{10}+\right. \\
& +\left(0.312301 \mu_{1}+1.006250 \nu_{1}+0.063901\right) y_{11}+ \\
& \left.\left.+\left(-0.392473 \mu_{1}-1.264569 \nu_{1}-0.370296\right) Y_{21}\right] \sin \frac{3}{2} \nu\right]+O\left(e^{5 / 2}\right) \\
q_{2} & =e^{1 / 2}\left[-\left(0.269671 \mu_{1}-0.646644 \nu_{1}+1.522315\right) \cos \frac{\nu}{2}+\right. \\
& \left.+\left(0.0185910 \mu_{1}+0.0599013 \nu_{1}-.208294\right) \cos \frac{3}{2} \nu\right] y_{10}+ \\
& +e^{3 / 2}\left[\left[\left(0.0679485 \mu_{1}+2.876580 \nu_{1}+0.390343\right) y_{10}+\right.\right. \\
& +\left(0.163368 \mu_{1}+2.041920 \nu_{1}-1.752728\right) y_{11}+ \\
& \left.-\left(0.812287 \mu_{1}+3.970059 \nu_{1}-1.400310\right) Y_{21}\right] \cos \frac{\nu}{2}+ \\
& +\left[-\left(0.178975 \mu_{1}+0.576669 \nu_{1}-0.347254\right) y_{10}+\right. \\
& +\left(0.0557731 \mu_{1}+0.179704 \nu_{1}-0.228078\right) y_{11}+ \\
& \left.\left.+\left(0.321776 \mu_{1}+1.036781 \nu_{1}+0.281380\right) Y_{21}\right] \cos \frac{3}{2} \nu\right]+O\left(e^{5 / 2}\right) .
\end{aligned}
$$

The periodic solution corresponding to the second pair of equilibria is in its structure the same, and the sine and cosine functions change places; $y_{10}$ is replaced with $y_{20}, y_{11}$ with $y_{21}$, and $Y_{21}$ with $Y_{11}$; also, the signs of some coefficients in terms with $\mu_{1}$ and $\nu_{1}$ and the values of some terms that do not contain these quantities are reversed.

According to the results of Section 6.2, in nonresonant cases the periodic solution (8.3), (8.4) is stable for most initial conditions and is formally stable; for the cases of fourth-order resonance it is formally stable. The second periodic solution described above is unstable.

If we neglect terms of order $e^{3 / 2}$ and higher in the formulae (8.3), (8.4) and in the expressions for the second solution, we obtain equations of closed curves in the plane of $q_{1}$, $q_{2}$, with a typical size of order $e^{1 / 2}$. These curves are symmetric relative to both coordinate axes and intersect each axis at two or four points. The form of the curves depends on the chosen values of $\mu_{1}, \nu_{1}$ from the region of existence of the solution in a neighborhood of the resonant point. A number of typical variants of these curves are shown in Figs. 5a-5f.

\subsection{Point 2}

Consider the $e^{2}$-neighborhood of the resonant point $P_{3}$, which is defined in (5.1) for $\varepsilon=e$. We first make the following linear normalizing change of variables:

$$
\begin{array}{ll}
q_{1}=-0.018664 p_{1}^{\prime}+0.972395 q_{2}^{\prime}, & q_{2}=1.030098 q_{1}^{\prime}-0.019772 p_{2}^{\prime}, \\
p_{1}=0.428495 q_{1}^{\prime}+1.020164 p_{2}^{\prime}, & p_{2}=0.963018 p_{1}^{\prime}+0.404492 q_{2}^{\prime},
\end{array}
$$

which reduces the form $H_{20}$ to

$$
H_{20}=\frac{1}{2}\left(p_{1}^{\prime 2}+p_{2}^{\prime 2}\right)+\frac{3}{2}\left(q_{1}^{\prime} p_{2}^{\prime}-p_{1}^{\prime} q_{2}^{\prime}\right)
$$




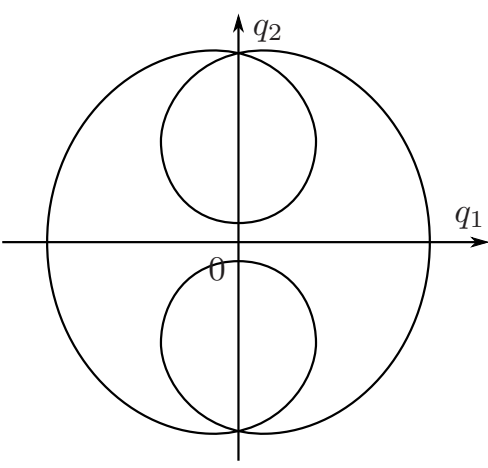

(a)

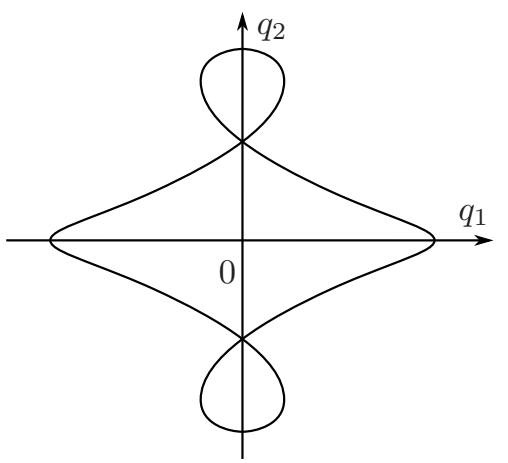

(d)

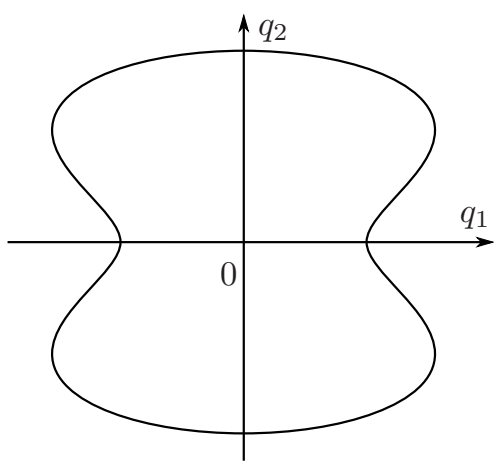

(b)

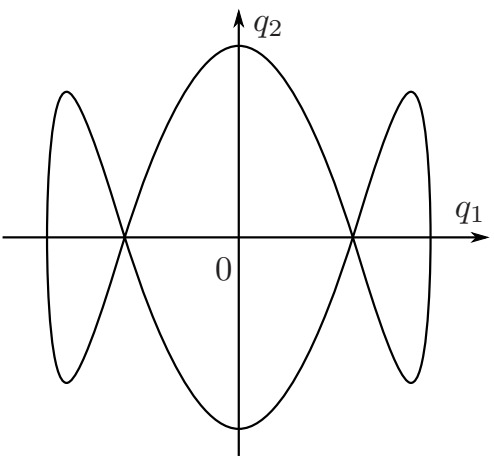

(e)

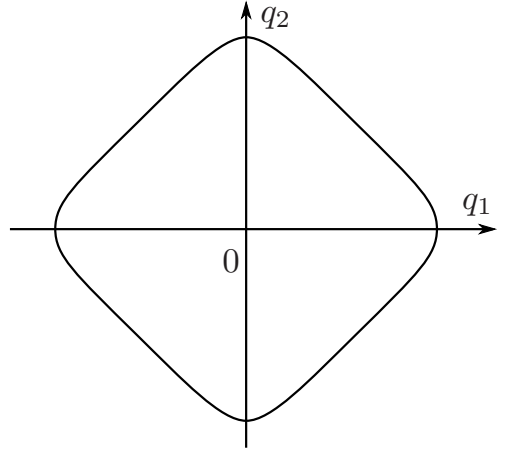

(c)

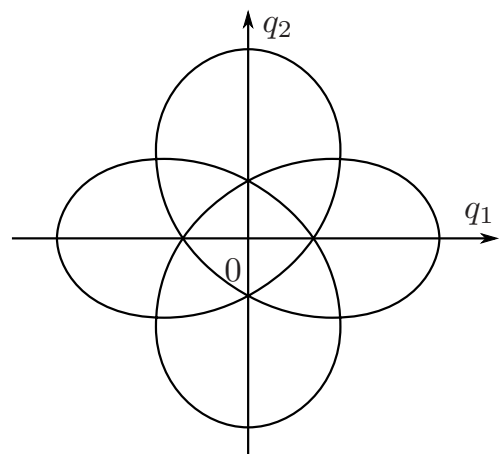

$(f)$

Fig. 5. Periodic motions in a neighborhood of point $P_{1}$.

and a normalization of the form $H_{40}$. This yields the expression (2.4) with the coefficients

$$
\gamma_{1}=0.060828, \quad \gamma_{2}=0.126671, \quad \gamma_{3}=0.168391 \text {. }
$$

Next, we transform the nonautonomous quadratic part in terms through order three in $e$. This gives $\Gamma_{21}=0$ and

$$
\begin{aligned}
\Gamma_{22} & =\left(-0.206325 \mu_{2}+1.012581 \nu_{2}-0.0444830\right)\left(y_{1}^{2}+y_{2}^{2}\right)+ \\
& +\left(-0.414942 \mu_{2}+0.637429 \nu_{2}-0.00205811\right)\left(y_{1} Y_{2}-Y_{1} y_{2}\right), \\
\Gamma_{23} & =\left(-0.206325 \mu_{3}+0.000880917\right) y_{1}^{2}+\left(-0.206325 \mu_{3}-0.000880917\right) y_{2}^{2}- \\
& -0.414942 \mu_{3}\left(y_{1} Y_{2}-Y_{1} y_{2}\right) .
\end{aligned}
$$

Let us introduce the notation

$$
\mu_{20}=-0.215597+4.907708 \nu_{2}, \quad \mu_{30}=-0.000426957 .
$$

It follows from the above relations that the conditions for stability of the trivial equilibrium of the system are given by the inequalities

$$
\mu_{2}<\mu_{20}, \quad \mu_{3}<\mu_{30} .
$$


When $\mu_{2}=\mu_{20}$, a model system of third approximation is considered for which the form $\Gamma_{22}$ becomes

$$
\Gamma_{22}=\left(0.0874022-1.398986 \nu_{2}\right)\left(y_{1} Y_{2}-Y_{1} y_{2}\right) .
$$

This system has isolated equilibrium points: for $\mu_{3}>\mu_{30}$, it has a linearly stable pair of equilibria given by

$$
\begin{gathered}
y_{1}=0, \quad Y_{2}=0, \quad y_{2}=y_{20}+e y_{21}+O\left(e^{2}\right), \quad Y_{1}=e^{2} Y_{12}+O\left(e^{3}\right), \\
y_{20}^{2}=0.00724101+1.695959 \mu_{3}, \quad Y_{12}=\left(0.0874022-1.398986 \nu_{2}\right) y_{20}, \\
y_{21}=\left(4.676218 \nu_{2}^{2}+2.100087 \nu_{2}-0.0327068 e\right) y_{20}^{-1},
\end{gathered}
$$

and for $\mu_{3}>-\mu_{30}$, it has an unstable pair of equilibria defined by the conditions

$$
\begin{gathered}
y_{2}=0, \quad Y_{1}=0, \quad y_{1}=y_{10}+e y_{11}+O\left(e^{2}\right), \quad Y_{2}=e^{2} Y_{22}+O\left(e^{3}\right), \\
y_{10}^{2}=-0.00724101+1.695959 \mu_{3}, \quad Y_{22}=(1.398986 \nu 2-0.0874022) y_{10}, \\
y_{11}=\left(4.676218 \nu_{2}^{2}+2.100087 \nu_{2}-0.0327068\right) y_{10}^{-1} .
\end{gathered}
$$

The periodic solution to the initial complete system, which is generated by the first pair of equilibria, can be represented as

$$
\begin{aligned}
q_{1} & =e^{3 / 2}\left[0.00888998 \sin \frac{\nu}{2}+0.972395 \sin \frac{3}{2} \nu-0.00615846 \sin \frac{5}{2} \nu\right] y_{10}+ \\
& +e^{5 / 2}\left[\left(0.104079 y_{10}+0.00888998 y_{11}+0.00161357 Y_{21}\right) \sin \frac{\nu}{2}+\right. \\
& +\left(0.972395 y_{11}+0.0186644 Y_{21}\right) \sin \frac{3}{2} \nu- \\
& \left.-\left(0.852579 y_{10}+0.00615846 y_{11}+0.00222669 Y_{21}\right) \sin \frac{5}{2} \nu\right]+O\left(e^{7 / 2}\right) \\
q_{2} & =e^{3 / 2}\left[-0.00555937 \cos \frac{\nu}{2}+1.030098 \cos \frac{3}{2} \nu+0.00845298 \cos \frac{5}{2} \nu\right] y_{10}+ \\
& +e^{5 / 2}\left[\left(0.143855 y_{10}-0.00555937 y_{11}-0.0177381 Y_{21}\right) \cos \frac{\nu}{2}+\right. \\
& +\left(1.030098 y_{11}-0.0197720 Y_{21}\right) \cos \frac{3}{2} \nu+ \\
& \left.+\left(-0.911498 y_{10}+0.00845298 y_{11}+0.00251863 Y_{21}\right) \cos \frac{5}{2} \nu\right]+O\left(e^{7 / 2}\right)
\end{aligned}
$$

The periodic solution generated by the second pair of equilibria is obtained if (as for point 1) in the above expressions we interchange the sine and cosine functions, replace $y_{10}$ with $y_{20}, y_{11}$ with $y_{21}$, and $Y_{21}$ with $Y_{11}$. In addition, in the expression for $q_{1}$ we need to reverse the signs in front of the terms with $Y_{11}$, and in the expression for $q_{2}$, the signs of all terms except for terms with $Y_{11}$.

The first of these periodic solutions is stable for most initial conditions and is formally stable in the absence of fourth-order resonances and is formally stable in resonant cases; the second periodic solution is unstable.

Let us neglect terms of order $e^{5 / 2}$ and higher in the solutions found above, and let us neglect in the remaining part terms with harmonics $\cos (\nu / 2), \sin (\nu / 2), \cos (5 \nu / 2), \sin (5 \nu / 2)$ in view of the smallness of the coefficients. As a result, we obtain equations of an ellipse, close to a circle, with semiaxes of order $e^{3 / 2}$. 


\section{References}

[1] Markeev, A. P., On a Multiple Resonance in Linear Hamiltonian Systems, Dokl. Phys., 2005, vol. 50, no. 5, pp. 278-282; see also: Dokl. Akad. Nauk, 2005, vol.402, no. 3, pp. 339-343.

[2] Markeyev, A.P., Multiple Parametric Resonance in Hamiltonian Systems, J. Appl. Math. Mech., 2006, vol.70, no. 2, pp. 176-194; see also: Prikl. Mat. Mekh., 2006, vol. 70, no. 2, pp. 200-220.

[3] Markeev, A. P., Linear Hamiltonian Systems and Some Problems on Stability of Motion of a Satellite about Its Center of Mass, Izhevsk: R\&C Dynamics, Institute of Computer Science, 2009 (Russian).

[4] Kholostova, O. V., On Periodic Motions of a Nonautonomous Hamiltonian System in One Case of Multiple Parametric Resonance, Nelin. Dinam., 2017, vol. 13, no. 4, pp. 477-504 (Russian).

[5] Kholostova, O. V., On Periodic Motions of a Nearly Autonomous Hamiltonian System in the Occurrence of Double Parametric Resonance, Mech. Solids, 2019, vol.54, no. 2, pp. 211-233; see also: Prikl. Mat. Mekh., 2019, vol. 83, no. 2, pp. 175-201.

[6] Kholostova, O. V., On the Motions of One Near-Autonomous Hamiltonian System at a $1: 1$ : 1 Resonance, Regul. Chaotic Dyn., 2019, vol.24, no.3, pp. 235-265.

[7] Kholostova, O. V., On the Motions of Near-Autonomous Hamiltonian System in the Cases of Two Zero Frequencies, Vestn. Udmurtsk. Univ. Mat. Mekh. Komp. Nauki, 2020, vol. 30, no. 4, pp.672-695 (Russian).

[8] Meyer, K. R. and Schmidt, D.S., Periodic Orbits near $\mathcal{L}_{4}$ for Mass Ratios near the Critical Mass Ratio of Routh, Celestial Mech., 1971, vol.4, no. 1, pp. 99-109.

[9] Schmidt, D. and Sweet, D., A Unifying Theory in Determining Periodic Families for Hamiltonian Systems at Resonance, J. Differential Equations, 1973, vol. 14, no. 3, pp. 597-609.

[10] Markeev, A. P., Libration Points in Celestial Mechanics and Space Dynamics, Moscow: Nauka, 1978 (Russian).

[11] Sokolsky, A.G., To the Problem on Stability of Regular Precessions of a Symmetrical Satellite, Kosmicheskie Issledovaniya, 1980, vol. 18, no. 5, pp. 698-706 (Russian).

[12] van der Meer, J.-C., Nonsemisimple 1 : 1 Resonance at an Equilibrium, Celestial Mech., 1982, vol. 27, no. 2, pp. 131-149.

[13] Treshchev, D. V., Loss of Stability in Hamiltonian Systems That Depend on Parameters, J. Appl. Math. Mech., 1992, vol.56, no.4, pp.492-500; see also: Prikl. Mat. Mekh., 1992, vol.56, no.4, pp. 587-596.

[14] Schmidt, D. S., Versal Normal Form of the Hamiltonian Function of the Restricted Problem of Three Bodies near $\mathcal{L}_{4}$, J. Comput. Appl. Math., 1994, vol.52, nos. 1-3, pp. 155-176.

[15] Bardin, B. S., On Motions near the Lagrange Equilibrium Point $\mathcal{L}_{4}$ in the Case of Routh's Critical Mass Ratio, Celestial Mech. Dynam. Astronom., 2002, vol. 82, no. 2, pp. 163-177.

[16] Lerman, L. M., and Markova, A. P., On Stability at the Hamiltonian Hopf Bifurcation, Regul. Chaotic Dyn., 2009, vol. 14, no. 1, pp. 148-162.

[17] Arnol'd, V.I., Kozlov, V. V., and Neřshtadt, A. I., Mathematical Aspects of Classical and Celestial Mechanics, 3rd ed., Encyclopaedia Math. Sci., vol. 3, Berlin: Springer, 2006.

[18] Malkin, I. G., Some Problems in the Theory of Nonlinear Oscillations: In 2 Vols.: Vol. 1, Germantown, Md.: United States Atomic Energy Commission, Technical Information Service, 1959.

[19] Glimm, J., Formal Stability of Hamiltonian Systems, Comm. Pure Appl. Math., 1964, vol. 17, no. 4, pp. 509-526.

[20] Markeev, A. P., On Rotational Motion of a Dynamically Symmetrical Satellite in an Elliptic Orbit, Kosmicheskie Issledovaniya, 1967, vol.5, no.4, pp. 530-539 (Russian).

[21] Beletskii, V.V., Satellite's Motion about Center of Mass in a Gravitational Field, Moscow: MGU, 1975 (Russian).

[22] Moser, J., Convergent Series Expansions for Quasi-Periodic Motions, Math. Ann., 1976, vol.169, no. 1, pp. 136-176.

[23] Mel'nikov, V. K., On Certain Cases of Conservation of Almost Periodic Motions with a Small Change of the Hamiltonian Function, Dokl. Akad. Nauk SSSR, 1965, vol. 165, no. 6, pp. 1245-1248 (Russian). 